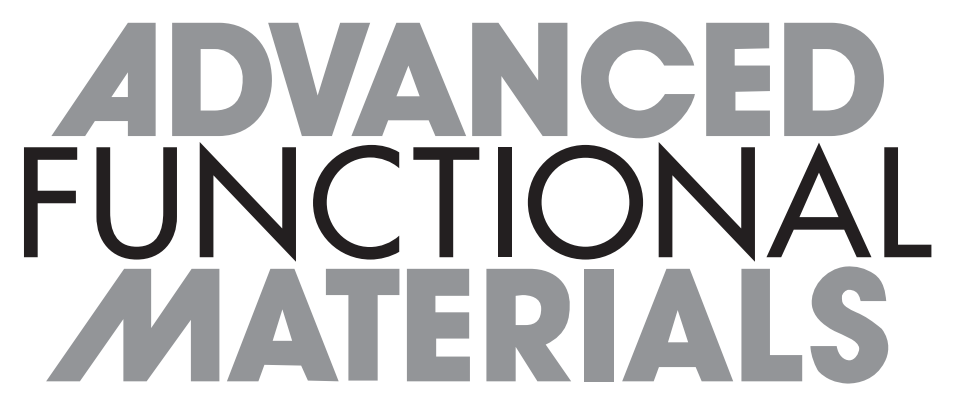

Supporting Information

for

(C) Wiley-VCH 2007

69451 Weinheim, Germany 


\section{Optical Power Limiters Based on Colorless Di-, Oligo- and Polymetallaynes. Highly Transparent Materials for Eye Protection Devices}


Synthesis and Characterization Data. In the shortform of each formula, the triple bonds are abbreviated by $\mathrm{T}$ and dioctylfluorene by $\mathrm{C}_{8}$-F. Model complexes and polymers are differentiated by use of the subscripts 1 and $\mathrm{n}$, respectively. The compounds $\left[\mathbf{H}-\mathbf{T}\left(\mathbf{C}_{\mathbf{8}}-\mathbf{F}\right) \mathbf{T}-\mathbf{H}\right]_{\mathbf{1}},\left[\mathbf{T}\left(\mathbf{C}_{\mathbf{8}}-\mathbf{F}\right) \mathbf{T}\right]_{\mathbf{n}}$, $\left[\mathbf{P t}-\mathbf{T}\left(\mathbf{C}_{8}-\mathbf{F}\right) \mathbf{T}-\mathbf{P t}\right]_{1}, \quad\left[\mathbf{H g}-\mathbf{T}\left(\mathbf{C}_{8}-\mathbf{F}\right) \mathbf{T}-\mathbf{H g}\right]_{1}, \quad\left[\mathbf{P t}-\mathbf{T}\left(\mathbf{C}_{8}-\mathbf{F}\right) \mathbf{T}\right]_{\mathbf{n}}$ and $\left[\mathbf{H g}-\mathbf{T}\left(\mathbf{C}_{8}-\mathbf{F}\right) \mathbf{T}\right]_{\mathbf{n}}$ were prepared according to the published procedures. ${ }^{[1,2]}$

\section{$\left[T\left(C_{8}-F\right) T-P t-T\left(C_{8}-F\right) T\right]_{1}$}

A solution of trans-[ $\left.\mathrm{PtCl}_{2}\left(\mathrm{PBu}_{3}\right)_{2}\right](0.153 \mathrm{~g}, 0.228 \mathrm{mmol})$ in $\mathrm{CH}_{2} \mathrm{Cl}_{2}(20 \mathrm{~mL})$ was added slowly to a mixture of excess $\left[\mathbf{H}-\mathbf{T}\left(\mathbf{C}_{\mathbf{8}} \mathbf{- F}\right) \mathbf{T}-\mathbf{H}\right]_{\mathbf{1}}(0.40 \mathrm{~g}, 0.913 \mathrm{mmol})$ and $\mathrm{CuI}(2 \mathrm{mg})$ in $\mathrm{NEt}_{3} / \mathrm{CH}_{2} \mathrm{Cl}_{2}(150 \mathrm{~mL}$, $1: 1, \mathrm{v} / \mathrm{v})$ at ambient temperature. After the addition, the reaction mixture was stirred at room temperature overnight. Following the filtration to remove the $\mathrm{CuI}$ catalyst residue, the filtrate was concentrated. The crude product was purified by column chromatography with hexane $/ \mathrm{CH}_{2} \mathrm{Cl}_{2}(3: 1$, $\mathrm{v} / \mathrm{v})$ as eluent. The product was obtained as an off-white crystalline solid $(0.235 \mathrm{~g}, 70 \%)$. IR (KBr): $v(\mathrm{C} \equiv \mathrm{C}) 2093 \mathrm{~cm}^{-1} .{ }^{1} \mathrm{H}$ NMR $\left(400 \mathrm{MHz}, \mathrm{CDCl}_{3}\right): \delta=7.55(\mathrm{~d}, J=7.6 \mathrm{~Hz}, 2 \mathrm{H}, \mathrm{Ar}), 7.51(\mathrm{~d}, J=8.0$

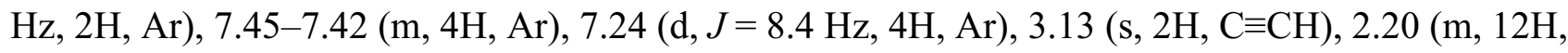
$\left.\mathrm{PBu}_{3}\right), 1.89\left(\mathrm{~m}, 8 \mathrm{H}, \mathrm{C}_{8} \mathrm{H}_{17}\right), 1.65\left(\mathrm{~m}, 12 \mathrm{H}, \mathrm{PBu}_{3}\right), 1.48\left(\mathrm{~m}, 12 \mathrm{H}, \mathrm{PBu}_{3}\right), 1.22-1.12\left(\mathrm{~m}, 40 \mathrm{H}, \mathrm{C}_{8} \mathrm{H}_{17}\right)$, $0.95\left(\mathrm{t}, J=7.2 \mathrm{~Hz}, 18 \mathrm{H}, \mathrm{PBu}_{3}\right), 0.83\left(\mathrm{t}, J=7.2 \mathrm{~Hz}, 12 \mathrm{H}, \mathrm{C}_{8} \mathrm{H}_{17}\right), 0.57 \mathrm{ppm}\left(\mathrm{br}, 8 \mathrm{H}, \mathrm{C}_{8} \mathrm{H}_{17}\right) .{ }^{13} \mathrm{C}$ NMR (100.3 MHz, $\left.\mathrm{CDCl}_{3}\right): \delta=150.53,141.92,137.12,130.97,129.62,128.11,126.23,125.09$, 
119.46, 119.23, 119.07 (Ar), 108.84, 100.45, 84.88, 76.72 (C $\equiv \mathrm{C}) 54.85$ (quat. C), 40.54, 31.83, 30.18, 29.36, 23.79, 22.66, $14.17\left(\mathrm{C}_{8} \mathrm{H}_{17}\right), 26.47,24.53,24.08,13.94 \mathrm{ppm}\left(\mathrm{PBu}_{3}\right) .{ }^{31} \mathrm{P}$ NMR $(161.9$ $\left.\mathrm{MHz}, \mathrm{CDCl}_{3}\right): \delta=4.02 \mathrm{ppm}\left({ }^{1} J_{\mathrm{P}-\mathrm{Pt}}=2348 \mathrm{~Hz}\right)$. FAB-MS: $m / z 1475[\mathrm{M}]^{+}$. Anal. Calcd for $\mathrm{C}_{90} \mathrm{H}_{136} \mathrm{P}_{2} \mathrm{Pt}: \mathrm{C}, 73.28 ; \mathrm{H}, 9.29$. Found: C, 73.12; H, 9.08.

\section{$\left[\mathrm{T}\left(\mathrm{C}_{8}-\mathrm{F}\right) \mathrm{T}-\mathrm{Hg}-\mathrm{T}\left(\mathrm{C}_{8}-\mathrm{F}\right) \mathrm{T}\right]_{1}$}

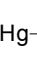

A basic solution of $\mathrm{NaOMe}(2.3 \mathrm{~mL}, 0.2 \mathrm{mmol} / \mathrm{L}$ in $\mathrm{MeOH})$ was added slowly to a solution of [H-T(C $\left.\mathbf{C}_{\mathbf{8}} \mathbf{- F}\right) \mathbf{T}-\mathrm{H}_{\mathbf{1}}(0.40 \mathrm{~g}, 0.913 \mathrm{mmol})$ in $\mathrm{MeOH} / \mathrm{CH}_{2} \mathrm{Cl}_{2}(150 \mathrm{~mL}, 10: 1, \mathrm{v} / \mathrm{v})$. Then, $\mathrm{HgCl}_{2}(0.062$ g, $0.229 \mathrm{mmol})$ in $\mathrm{MeOH}(15 \mathrm{~mL})$ was added dropwisely to the as-prepared solution. The reaction mixture turned milky. After stirring overnight at ambient temperature, the precipitate formed was collected and purified by preparative TLC plates with hexane $/ \mathrm{CH}_{2} \mathrm{Cl}_{2}(3: 1, \mathrm{v} / \mathrm{v})$ as eluent. The desired product was obtained as a pale yellow oil $(0.148 \mathrm{~g}, 60 \%)$. IR $(\mathrm{KBr}): v(\mathrm{C} \equiv \mathrm{C}) 2140 \mathrm{~cm}^{-1} .{ }^{1} \mathrm{H}$ NMR (400 MHz, $\left.\mathrm{CDCl}_{3}\right): \delta=7.61(\mathrm{~d}, J=7.2 \mathrm{~Hz}, 4 \mathrm{H}, \mathrm{Ar}), 7.50-7.47(\mathrm{~m}, 8 \mathrm{H}, \mathrm{Ar}), 3.15(\mathrm{~s}, 2 \mathrm{H}$, $\mathrm{C} \equiv \mathrm{CH}), 1.95\left(\mathrm{~m}, 8 \mathrm{H}, \mathrm{C}_{8} \mathrm{H}_{17}\right), 1.22-1.03\left(\mathrm{~m}, 40 \mathrm{H}, \mathrm{C}_{8} \mathrm{H}_{17}\right), 0.83\left(\mathrm{t}, J=7.2 \mathrm{~Hz}, 12 \mathrm{H}, \mathrm{C}_{8} \mathrm{H}_{17}\right), 0.59 \mathrm{ppm}$ (br, 8H, $\left.\mathrm{C}_{8} \mathrm{H}_{17}\right) .{ }^{13} \mathrm{C}$ NMR (100.3 MHz, $\left.\mathrm{CDCl}_{3}\right): \delta=151.07,151.04,140.93,131.49,131.22$, $126.70,126.49,121.01,120.84,120.80,120.02,119.97(\mathrm{Ar}), 106.88,84.50,77.38(\mathrm{C} \equiv \mathrm{C}) 55.17$ (quat. C), 40.22, 31.37, 29.94, 29.20, 23.65, 22.56, 14.08 ppm $\left(\mathrm{C}_{8} \mathrm{H}_{17}\right)$. FAB-MS: $m / z 1075$ [M] $]^{+}$ Anal. Calcd (\%) for $\mathrm{C}_{66} \mathrm{H}_{82} \mathrm{Hg}$ : C, 73.68; H, 7.68. Found: C, 73.54; H, 7.48.

\section{$\left[\mathrm{Au}-\mathrm{T}\left(\mathrm{C}_{8}-\mathrm{F}\right) \mathrm{T}-\mathrm{Au}\right]_{1}$}




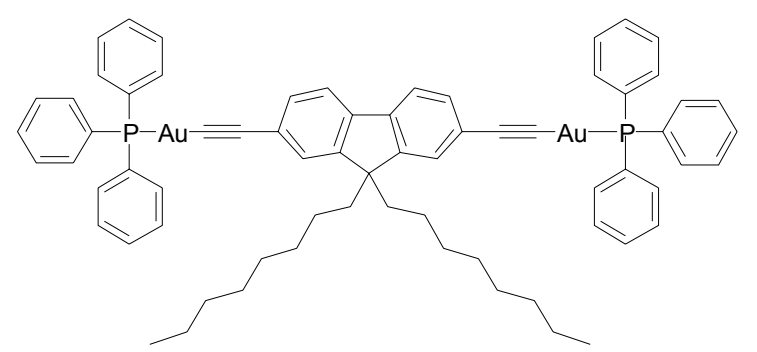

To a solution mixture of $\left[\mathbf{H}-\mathbf{T}\left(\mathbf{C}_{\mathbf{8}} \mathbf{- F}\right) \mathbf{T}-\mathbf{H}\right]_{1}(0.10 \mathrm{~g}, 0.228 \mathrm{mmol})$ and $\mathrm{Au}\left(\mathrm{PPh}_{3}\right) \mathrm{Cl}(0.25 \mathrm{~g}, 0.502$ mmol) in $\mathrm{MeOH}(50 \mathrm{~mL}), \mathrm{NaOMe}$ solution $(2.5 \mathrm{~mL}, 0.2 \mathrm{mmol} / \mathrm{L}$ in $\mathrm{MeOH})$ was added with a syringe. The reaction mixture was stirred overnight. The white precipitate was collected and washed with $\mathrm{MeOH}$. Then, the product was purified by reprecipitating its concentrated $\mathrm{CH}_{2} \mathrm{Cl}_{2}$ solution from $\mathrm{MeOH}$. The product as a white solid was collected and dried under vacuum $(0.236 \mathrm{~g}, 77 \%)$. IR $(\mathrm{KBr}): v(\mathrm{C} \equiv \mathrm{C}) 2100 \mathrm{~cm}^{-1} .{ }^{1} \mathrm{H}$ NMR $\left(400 \mathrm{MHz}, \mathrm{CDCl}_{3}\right): \delta=7.59-7.43(\mathrm{~m}, 36 \mathrm{H}, \mathrm{Ar}), 1.87$ (m, 4H, $\left.\mathrm{C}_{8} \mathrm{H}_{17}\right), 1.22-0.97\left(\mathrm{~m}, 20 \mathrm{H}, \mathrm{C}_{8} \mathrm{H}_{17}\right), 0.81\left(\mathrm{t}, J=7.2 \mathrm{~Hz}, 6 \mathrm{H}, \mathrm{C}_{8} \mathrm{H}_{17}\right), 0.53 \mathrm{ppm}\left(\mathrm{br}, 4 \mathrm{H}, \mathrm{C}_{8} \mathrm{H}_{17}\right) .{ }^{13} \mathrm{C}$ NMR (100.3 MHz, $\left.\mathrm{CDCl}_{3}\right): \delta=150.61,139.76,134.24,131.52,131.04,130.01,129.46,129.06$, 126.96, $122.82(\mathrm{Ar}), 119.28,105.32(\mathrm{C} \equiv \mathrm{C}) 54.84$ (quat. C), 40.63, 31.74, 30.16, 29.34, 29.32, 23.70, 22.54, 14.09 ppm $\left(\mathrm{C}_{8} \mathrm{H}_{17}\right) .{ }^{31} \mathrm{P}$ NMR (161.9 MHz, $\left.\mathrm{CDCl}_{3}\right): \delta=43.42$ ppm. FAB-MS: $\mathrm{m} / z 1356[\mathrm{M}]^{+}$. Anal. Calcd (\%) for $\mathrm{C}_{69} \mathrm{H}_{70} \mathrm{Au}_{2} \mathrm{P}_{2}$ : C, 61.15; H, 5.21. Found: C, 60.98; H, 5.04.

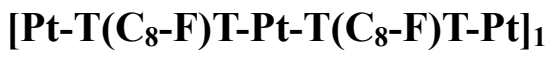

[T(C $\mathbf{C}_{\mathbf{8}}$-F)T-Pt-T(C $\left.\left.\mathbf{8}-\mathbf{F}\right) \mathbf{T}\right]_{1}(0.15 \mathrm{~g}, 0.102 \mathrm{mmol})$ and trans-[PtCl$\left.(\mathrm{Ph})\left(\mathrm{PEt}_{3}\right)_{2}\right](0.12 \mathrm{~g}, 0.221 \mathrm{mmol})$ were dissolved in $\mathrm{NEt}_{3} / \mathrm{CH}_{2} \mathrm{Cl}_{2}(50 \mathrm{~mL}, 1: 1 \mathrm{v} / \mathrm{v})$ at room temperature and a small amount of $\mathrm{CuI}$ was 
then added. After the reaction mixture was stirred at room temperature overnight, it was filtered through a short silica gel pad. The filtrate was concentrated and the residue was separated by preparative TLC eluting with hexane/ $\mathrm{CH}_{2} \mathrm{Cl}_{2}(2: 1, \mathrm{v} / \mathrm{v})$. The product was obtained as a viscous oil $(0.19 \mathrm{~g}, 75 \%)$. IR (KBr): $v(\mathrm{C} \equiv \mathrm{C}) 2096 \mathrm{~cm}^{-1} .{ }^{1} \mathrm{H}$ NMR $\left(400 \mathrm{MHz}, \mathrm{CDCl}_{3}\right): \delta=7.46(\mathrm{~d}, J=3.2 \mathrm{~Hz}$, 2H, Ar), 7.40 (d, $J=2.8 \mathrm{~Hz}, 2 \mathrm{H}, \mathrm{Ar}), 7.34$ (d, $J=7.2 \mathrm{~Hz}, 4 \mathrm{H}, \mathrm{Ar}), 7.25-7.20$ (m, 8H, Ar), 6.97 (t, $J$ $=7.6 \mathrm{~Hz}, 4 \mathrm{H}, \mathrm{Ar}), 6.82(\mathrm{t}, J=7.2 \mathrm{~Hz}, 2 \mathrm{H}, \mathrm{Ar}), 2.22-2.18\left(\mathrm{~m}, 12 \mathrm{H}, \mathrm{PBu}_{3}\right.$ and $\left.\mathrm{C}_{8} \mathrm{H}_{17}\right), 1.89-1.75(\mathrm{~m}$, $32 \mathrm{H}, \mathrm{PEt}_{3}$ and $\left.\mathrm{C}_{8} \mathrm{H}_{17}\right), 1.67-1.64\left(\mathrm{~m}, 12 \mathrm{H}, \mathrm{PBu}_{3}\right), 1.50-1.45\left(\mathrm{~m}, 12 \mathrm{H}, \mathrm{PBu}_{3}\right), 1.25-1.00(\mathrm{~m}, 76 \mathrm{H}$,

$\mathrm{PEt}_{3}$ and $\left.\mathrm{C}_{8} \mathrm{H}_{17}\right), 0.95\left(\mathrm{t}, J=7.2 \mathrm{~Hz}, 18 \mathrm{H}, \mathrm{PBu}_{3}\right), 0.83\left(\mathrm{t}, J=7.2 \mathrm{~Hz}, 12 \mathrm{H}, \mathrm{C}_{8} \mathrm{H}_{17}\right), 0.65 \mathrm{ppm}(\mathrm{br}, 8 \mathrm{H}$, $\left.\mathrm{C}_{8} \mathrm{H}_{17}\right) .{ }^{13} \mathrm{C} \mathrm{NMR}\left(100.3 \mathrm{MHz}, \mathrm{CDCl}_{3}\right): \delta=156.43,150.30,139.15,138.32,138.09,136.74,129.68$, $129.48,127.83,127.25,127.15,126.78,125.23,125.14,121.19,118.72$ (Ar), 112.84, 111.58, 110.31, $107.67(\mathrm{C} \equiv \mathrm{C}) 54.43$ (quat. C), 40.63, 31.80, 30.25, 29.39, 29.35, 23.75, 22.57, $13.85\left(\mathrm{C}_{8} \mathrm{H}_{17}\right), 26.36$, 24.45, 23.93, $14.08\left(\mathrm{PBu}_{3}\right), 15.12,7.78 \mathrm{ppm}\left(\mathrm{PEt}_{3}\right) .{ }^{31} \mathrm{P} \mathrm{NMR}\left(161.9 \mathrm{MHz}, \mathrm{CDCl}_{3}\right): \delta=10.70\left({ }^{1} J_{\mathrm{P}-\mathrm{Pt}}\right.$ $\left.=2638 \mathrm{~Hz}, \mathrm{PEt}_{3}\right), 3.81 \mathrm{ppm}\left({ }^{1} J_{\mathrm{P}-\mathrm{Pt}}=2353 \mathrm{~Hz}, \mathrm{PBu}_{3}\right)$. FAB-MS: $m / z 2490[\mathrm{M}]^{+}$. Anal. Calcd $(\%)$ for $\mathrm{C}_{126} \mathrm{H}_{204} \mathrm{P}_{6} \mathrm{Pt}_{3}: \mathrm{C}, 60.78 ; \mathrm{H}, 8.26$; Found: $\mathrm{C}, 60.55 ; \mathrm{H}, 8.02$.

\section{$\left[\mathrm{Hg}-\mathrm{T}\left(\mathrm{C}_{8}-\mathrm{F}\right) \mathrm{T}-\mathrm{Hg}-\mathrm{T}\left(\mathrm{C}_{8}-\mathrm{F}\right) \mathrm{T}-\mathrm{Hg}\right]_{1}$}

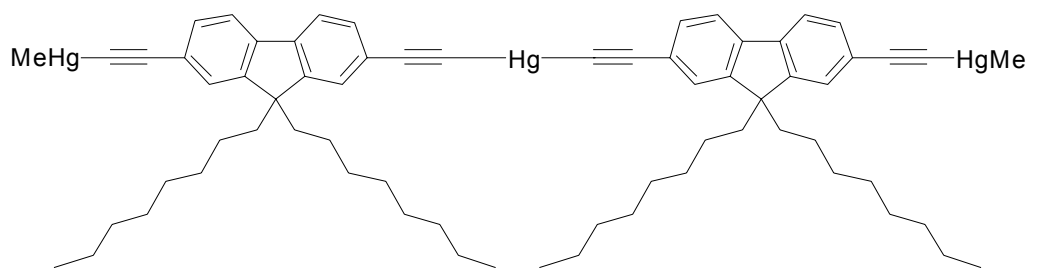

To a mixture of $\left[\mathbf{T}\left(\mathbf{C}_{\mathbf{8}} \mathbf{- F}\right) \mathbf{T}-\mathbf{H g}-\mathbf{T}\left(\mathbf{C}_{\mathbf{8}}-\mathbf{F}\right) \mathbf{T}\right]_{\mathbf{1}}(0.12 \mathrm{~g}, 0.112 \mathrm{mmol})$ and $\mathrm{MeHgCl}(0.070 \mathrm{~g}, 0.279 \mathrm{mmol})$ in $\mathrm{CH}_{2} \mathrm{Cl}_{2} / \mathrm{MeOH}(50 \mathrm{~mL}, 50: 1, \mathrm{v} / \mathrm{v}), \mathrm{NaOMe}$ solution $(1.5 \mathrm{~mL}, 0.2 \mathrm{mmol} / \mathrm{L}$ in $\mathrm{MeOH})$ was added. The reaction mixture was stirred overnight after which time the reaction mixture was concentrated 
and the residue was purified by preparative TLC plates using hexane/ $\mathrm{CH}_{2} \mathrm{Cl}_{2}(2: 1, \mathrm{v} / \mathrm{v})$ as eluent. The product was obtained as a white solid after drying under vacuum $(0.116 \mathrm{~g}, 69 \%)$. IR (KBr): $v(\mathrm{C} \equiv \mathrm{C}) 2123 \mathrm{~cm}^{-1} .{ }^{1} \mathrm{H}$ NMR $\left(400 \mathrm{MHz}, \mathrm{CDCl}_{3}\right): \delta=7.57(\mathrm{~d}, J=8.4 \mathrm{~Hz}, 4 \mathrm{H}, \mathrm{Ar}), 7.45-7.42(\mathrm{~m}, 8 \mathrm{H}$, Ar), 1.94-1.88 (m, 8H, $\left.\mathrm{C}_{8} \mathrm{H}_{17}\right), 1.25-1.01\left(\mathrm{~m}, 40 \mathrm{H}, \mathrm{C}_{8} \mathrm{H}_{17}\right), 0.82$ (t, J=7.0 Hz, $\left.12 \mathrm{H}, \mathrm{C}_{8} \mathrm{H}_{17}\right), 0.70$ (s, 6H, HgMe), 0.55 ppm (br, 8H, $\mathrm{C}_{8} \mathrm{H}_{17}$ ). FAB-MS: $m / z 1505[\mathrm{M}]^{+}$. Anal. Calcd (\%) for $\mathrm{C}_{68} \mathrm{H}_{86} \mathrm{Hg}_{3}$ : C, 54.26; H, 5.76. Found: C, 54.14; H, 5.43.

\section{$\left[P t-T\left(C_{8}-F\right) T-H g-T\left(C_{8}-F\right) T-P t\right]_{1}$}

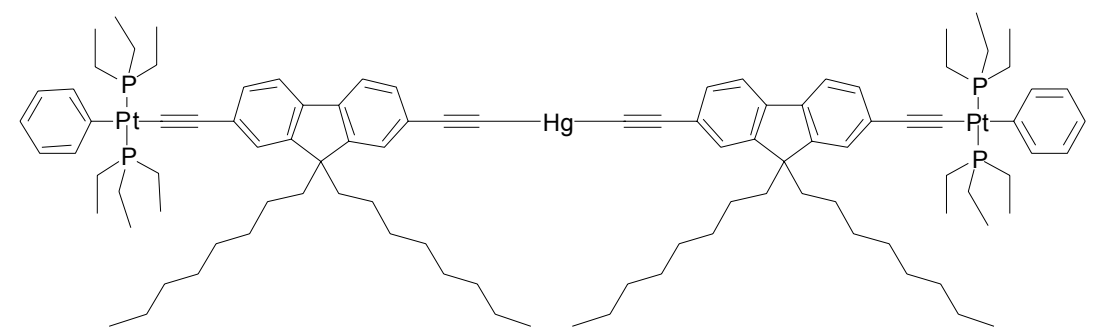

$\left[\mathbf{T}\left(\mathbf{C}_{\mathbf{8}} \mathbf{- F}\right) \mathbf{T}-\mathbf{H g}-\mathbf{T}\left(\mathbf{C}_{\mathbf{8}} \mathbf{- F}\right) \mathbf{T}\right]_{1}(0.10 \mathrm{~g}, 0.0929 \mathrm{mmol})$ and trans-[PtCl$\left.(\mathrm{Ph})\left(\mathrm{PEt}_{3}\right)_{2}\right](0.126 \mathrm{~g}, 0.232 \mathrm{mmol})$ were added to a mixture of $\mathrm{NEt}_{3} / \mathrm{CH}_{2} \mathrm{Cl}_{2}(40 \mathrm{~mL}, 1: 1 \mathrm{v} / \mathrm{v})$ at room temperature and a small amount of $\mathrm{CuI}$ was added as the catalyst. After the reaction mixture was stirred at ambient temperature overnight, it was filtered through a short pad of silica gel to remove the catalyst. The solvent was removed under reduced pressure and the crude product was separated by preparative TLC plates with hexane/ $\mathrm{CH}_{2} \mathrm{Cl}_{2}(3: 1, \mathrm{v} / \mathrm{v})$ as eluent. The product obtained from the first-round separation was separated again with preparative TLC plates using hexane/ethyl acetate $(24: 1, \mathrm{v} / \mathrm{v})$ as eluent. In this way, the title product in a pure form was obtained as viscous oil $(0.122 \mathrm{~g}, 61 \%)$. IR $(\mathrm{KBr}): v(\mathrm{C} \equiv \mathrm{C})$ $2089 \mathrm{~cm}^{-1} .{ }^{1} \mathrm{H}$ NMR $\left(400 \mathrm{MHz}, \mathrm{CDCl}_{3}\right): \delta=7.56(\mathrm{~d}, J=7.6 \mathrm{~Hz}, 2 \mathrm{H}, \mathrm{Ar}), 7.50(\mathrm{~d}, J=8.0 \mathrm{~Hz}, 2 \mathrm{H}$, Ar), 7.46-7.43 (m, 4H, Ar), 7.36-7.25 (m, 8H, Ar), $6.98(\mathrm{t}, J=7.6 \mathrm{~Hz}, 4 \mathrm{H}, \mathrm{Ar}), 6.82$ (t, $J=7.6 \mathrm{~Hz}$, 2H, Ar), $1.90\left(\mathrm{t}, J=8.4 \mathrm{~Hz}, 8 \mathrm{H}, \mathrm{C}_{8} \mathrm{H}_{17}\right), 1.83-1.76\left(\mathrm{~m}, 24 \mathrm{H}, \mathrm{PEt}_{3}\right), 1.28-0.88\left(\mathrm{~m}, 76 \mathrm{H}, \mathrm{PEt}_{3}\right.$ and 
$\left.\mathrm{C}_{8} \mathrm{H}_{17}\right), 0.83\left(\mathrm{t}, J=7.2 \mathrm{~Hz}, 12 \mathrm{H}, \mathrm{C}_{8} \mathrm{H}_{17}\right), 0.63 \mathrm{ppm}$ (br, 8H, $\left.\mathrm{C}_{8} \mathrm{H}_{17}\right) .{ }^{13} \mathrm{C} \mathrm{NMR}\left(100.3 \mathrm{MHz}, \mathrm{CDCl}_{3}\right)$ : $\delta=156.23,150.77,142.11,139.11,136.97,131.33,129.92,128.56,127.31,126.55,125.31,121.27$, 120.33, 119.53, 119.27, 119.21 (Ar), 114.44, 111.48, 107.30 (C $\equiv \mathrm{C}) 54.79$ (quat. C), 40.37, 31.78, 30.10, 29.30, 29.29, 23.71, 22.58, $14.10\left(\mathrm{C}_{8} \mathrm{H}_{17}\right), 15.05,8.06 \mathrm{ppm}\left(\mathrm{PEt}_{3}\right) .{ }^{31} \mathrm{P}$ NMR $(161.9 \mathrm{MHz}$, $\left.\mathrm{CDCl}_{3}\right): \delta=10.73 \mathrm{ppm}\left({ }^{1} J_{\mathrm{P}-\mathrm{Pt}}=2634 \mathrm{~Hz}, \mathrm{PtPEt}_{3}\right)$. FAB-MS: $m / z 2090[\mathrm{M}]^{+}$. Anal. Calcd (\%) for $\mathrm{C}_{102} \mathrm{H}_{150} \mathrm{HgP}_{4} \mathrm{Pt}_{2}$ : C, 58.59; H, 7.23. Found: C, 58.40; H, 7.10.

\section{$\left[\mathrm{Hg}-\mathrm{T}\left(\mathrm{C}_{8}-\mathrm{F}\right) \mathrm{T}-\mathrm{Pt}-\mathrm{T}\left(\mathrm{C}_{8}-\mathrm{F}\right) \mathrm{T}-\mathrm{Hg}\right]_{1}$}

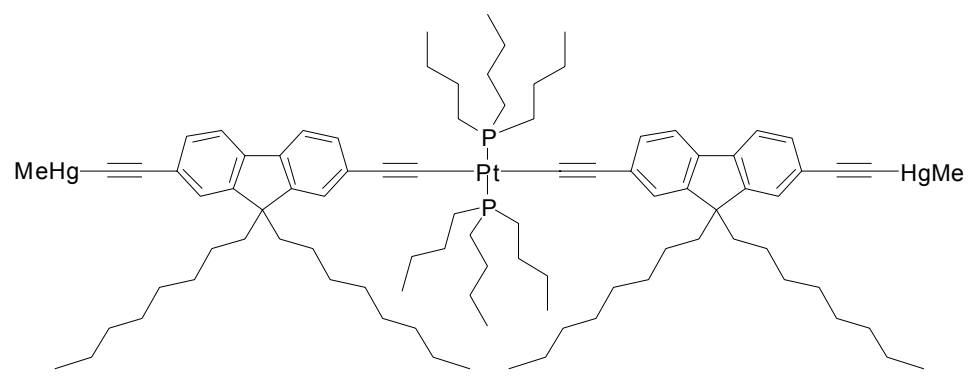

The metal precursor $\left[\mathbf{T}\left(\mathbf{C}_{\mathbf{8}} \mathbf{- F}\right) \mathbf{T}-\mathbf{P t}-\mathbf{T}\left(\mathbf{C}_{\mathbf{8}} \mathbf{- F}\right) \mathbf{T}\right]_{\mathbf{1}}(0.100 \mathrm{~g}, 0.068 \mathrm{mmol})$ and $\mathrm{MeHgCl}(0.043 \mathrm{~g}, 0.169$ mmol) were mixed in $\mathrm{MeOH} / \mathrm{CH}_{2} \mathrm{Cl}_{2}(40 \mathrm{~mL}, 1: 40, \mathrm{v} / \mathrm{v})$ to form a clear solution. Then, a basic NaOMe solution $(1.0 \mathrm{~mL}, 0.2 \mathrm{mmol} / \mathrm{L}$ in $\mathrm{MeOH})$ was added to the reaction mixture at room temperature. After stirring the mixture overnight at ambient temperature, it was filtered and the filtrate was allowed to evaporate slowly to form almost colorless crystals of $\left[\mathbf{H g}-\mathbf{T}\left(\mathbf{C}_{\mathbf{8}}-\mathbf{F}\right) \mathbf{T}-\mathbf{P t}-\mathbf{T}\left(\mathbf{C}_{\mathbf{8}}-\mathbf{F}\right) \mathbf{T}-\mathbf{H g}\right]_{1}$ which were then collected and further recrystallized from a $\mathrm{CH}_{2} \mathrm{Cl}_{2} / \mathrm{MeOH}$ solution (0.085 g, 66\%). IR (KBr): $v(\mathrm{C} \equiv \mathrm{C}) 2089 \mathrm{~cm}^{-1} .{ }^{1} \mathrm{H}$ NMR $\left(400 \mathrm{MHz}, \mathrm{CDCl}_{3}\right)$ : $\delta=7.53(\mathrm{~d}, J=7.6 \mathrm{~Hz}, 2 \mathrm{H}, \mathrm{Ar}), 7.49(\mathrm{~d}, J=8.0 \mathrm{~Hz}, 2 \mathrm{H}, \mathrm{Ar}), 7.42-7.40(\mathrm{~m}, 4 \mathrm{H}, \mathrm{Ar}), 7.22(\mathrm{~d}, J=7.6$ $\mathrm{Hz}, 4 \mathrm{H}, \mathrm{Ar}), 2.19(\mathrm{~m}, 12 \mathrm{H}, \mathrm{Bu}), 1.87\left(\mathrm{~m}, 8 \mathrm{H}, \mathrm{C}_{8} \mathrm{H}_{17}\right), 1.65(\mathrm{~m} \mathrm{12H}, \mathrm{Bu}), 1.46(\mathrm{~m}, 12 \mathrm{H}, \mathrm{Bu})$, 1.21-0.94 (m, 58H, Bu and $\left.\mathrm{C}_{8} \mathrm{H}_{17}\right), 0.82\left(\mathrm{t}, J=7.2 \mathrm{~Hz}, 12 \mathrm{H}, \mathrm{C}_{8} \mathrm{H}_{17}\right), 0.70(\mathrm{~s}, 6 \mathrm{H}, \mathrm{HgMe}), 0.57 \mathrm{ppm}$ 
(br, $\left.8 \mathrm{H}, \mathrm{C}_{8} \mathrm{H}_{17}\right) .{ }^{13} \mathrm{C}$ NMR (100.3 MHz, $\left.\mathrm{CDCl}_{3}\right): \delta=150.64,142.46,141.44,137.41,131.03,129.67$, 127.99, 126.38, 125.16, 120.35, 119.44, 119.20 (Ar), 110.24, 108.77, 106.73 (C $\equiv \mathrm{C}) 54.77$ (quat. C), 40.57, 31.78, 30.17, 29.34, 23.73, 22.57, $14.08\left(\mathrm{C}_{8} \mathrm{H}_{17}\right), 26.37,24.45,23.96,13.84(\mathrm{Bu}), 7.14$ ppm (HgMe). ${ }^{31} \mathrm{P}$ NMR (161.9 MHz, $\left.\mathrm{CDCl}_{3}\right): \delta=3.92 \mathrm{ppm}\left({ }^{1} J_{\mathrm{P}-\mathrm{Pt}}=2350 \mathrm{~Hz}\right)$. FAB-MS: $m / z 1904[\mathrm{M}]^{+}$. Anal. Calcd (\%) for $\mathrm{C}_{92} \mathrm{H}_{140} \mathrm{Hg}_{2} \mathrm{P}_{2} \mathrm{Pt}$ : C, 58.03; H, 7.41. Found: C, 57.88; H, 7.10.

\section{$\left[\mathrm{Au}-\mathrm{T}\left(\mathrm{C}_{8}-\mathrm{F}\right) \mathrm{T}-\mathrm{Hg}-\mathrm{T}\left(\mathrm{C}_{8}-\mathrm{F}\right) \mathrm{T}-\mathrm{Au}\right]_{1}$}

$\left[\mathbf{T}\left(\mathbf{C}_{\mathbf{8}} \mathbf{- F}\right) \mathbf{T}-\mathbf{H g}-\mathbf{T}\left(\mathbf{C}_{\mathbf{8}} \mathbf{- F}\right) \mathbf{T}\right]_{1}(0.12 \mathrm{~g}, 0.112 \mathrm{mmol})$ and $\mathrm{Au}\left(\mathrm{PPh}_{3}\right) \mathrm{Cl}(0.138 \mathrm{~g}, 0.279 \mathrm{mmol})$ were combined in $\mathrm{MeOH} / \mathrm{CH}_{2} \mathrm{Cl}_{2}(45 \mathrm{~mL}, 3: 2 \mathrm{v} / \mathrm{v})$ to form a clear solution. Then, basic $\mathrm{NaOH}$ solution $(1.5 \mathrm{~mL}, 0.2 \mathrm{mmol} / \mathrm{L}$ in $\mathrm{MeOH})$ was added to the reaction mixture at room temperature. After the reaction proceeded overnight at ambient temperature, the reaction mixture was concentrated. The residue was dissolved in a small amount of $\mathrm{CH}_{2} \mathrm{Cl}_{2}(8 \mathrm{~mL})$ and poured into $\mathrm{MeOH}(50 \mathrm{~mL})$. The operation was repeated three times. The pale yellow precipitate was collected by filtration and dried under vacuum $(0.142 \mathrm{~g}, 64 \%)$. IR $(\mathrm{KBr}): v(\mathrm{C} \equiv \mathrm{C}) 2140 \mathrm{~cm}^{-1} \cdot{ }^{1} \mathrm{H}$ NMR $\left(400 \mathrm{MHz}, \mathrm{CDCl}_{3}\right): \delta=$ 7.60-7.48 (m, 42H, Ar), $1.91\left(\mathrm{~m}, 8 \mathrm{H}, \mathrm{C}_{8} \mathrm{H}_{17}\right), 1.23-1.01\left(\mathrm{~m}, 40 \mathrm{H}, \mathrm{C}_{8} \mathrm{H}_{17}\right), 0.82(\mathrm{t}, J=9.6 \mathrm{~Hz}, 12 \mathrm{H}$, $\left.\mathrm{C}_{8} \mathrm{H}_{17}\right), 0.55 \mathrm{ppm}\left(\mathrm{br}, 8 \mathrm{H}, \mathrm{C}_{8} \mathrm{H}_{17}\right) .{ }^{13} \mathrm{C} \mathrm{NMR}\left(100.3 \mathrm{MHz}, \mathrm{CDCl}_{3}\right): \delta=151.05,150.70,139.76$, $134.23,131.55,131.17,129.95,129.40,129.07,127.06,126.56,120.82,120.60,119.70,119.63$, 119.29 (Ar), 107.14, 105.36, 84.77 (C $\equiv$ C) 55.01 (quat. C), 40.45, 31.74, 30.16, 30.04, 29.27, 23.69, 22.56, 14.09 ppm $\left(\mathrm{C}_{8} \mathrm{H}_{17}\right) .{ }^{31} \mathrm{P}$ NMR (161.9 MHz, $\left.\mathrm{CDCl}_{3}\right): \delta=43.39$ ppm. FAB-MS: $m / z 1993[\mathrm{M}]^{+}$. 
Anal. Calcd (\%) for $\mathrm{C}_{102} \mathrm{H}_{110} \mathrm{Au}_{2} \mathrm{HgP}_{2}$ : C, 61.49; H, 5.56. Found: C, 61.21; H, 5.34.

\section{$\left[\mathrm{Au}-\mathrm{T}\left(\mathrm{C}_{8}-\mathrm{F}\right) \mathrm{T}-\mathrm{Pt}-\mathrm{T}\left(\mathrm{C}_{8}-\mathrm{F}\right) \mathrm{T}-\mathrm{Au}\right]_{1}$}

[T(C $\mathbf{C}_{\mathbf{8}}$-F)T-Pt-T(C $\left.\left.\mathbf{8}-\mathbf{F}\right) \mathbf{T}\right]_{1}(0.11 \mathrm{~g}, 0.075 \mathrm{mmol})$ and $\mathrm{Au}\left(\mathrm{PPh}_{3}\right) \mathrm{Cl}(0.093 \mathrm{~g}, 0.186 \mathrm{mmol})$ were added to a mixture of $\mathrm{MeOH} / \mathrm{CH}_{2} \mathrm{Cl}_{2}(45 \mathrm{~mL}, 3: 2 \mathrm{v} / \mathrm{v})$ to form a clear solution. NaOMe solution (1.1 mL, $0.2 \mathrm{mmol} / \mathrm{L}$ in $\mathrm{MeOH}$ ) was added to the reaction mixture at room temperature. After the reaction proceeded at ambient temperature overnight, the reaction mixture was concentrated. The residue was dissolved in a small amount of $\mathrm{CH}_{2} \mathrm{Cl}_{2}(6 \mathrm{~mL})$ and poured into $\mathrm{MeOH}(40 \mathrm{~mL})$. The operation was repeated three times. The pale yellow precipitate was collected by filtration and dried under vacuum (0.12 g, 63\%). IR (KBr): $v(\mathrm{C} \equiv \mathrm{C}) 2092 \mathrm{~cm}^{-1} .{ }^{1} \mathrm{H}$ NMR (400 MHz, $\left.\mathrm{CDCl}_{3}\right): \delta=7.60-7.48(\mathrm{~m}, 38 \mathrm{H}$, Ar), 7.23-7.21 (m, 4H, Ar), $2.20\left(\mathrm{~m}, 12 \mathrm{H}, \mathrm{PBu}_{3}\right), 1.90-1.86\left(\mathrm{~m}, 8 \mathrm{H}, \mathrm{C}_{8} \mathrm{H}_{17}\right), 1.67$ (br, 12H, $\left.\mathrm{PBu}_{3}\right)$, $1.50-1.46\left(\mathrm{~m}, 12 \mathrm{H}, \mathrm{PBu}_{3}\right), 1.20-0.91\left(\mathrm{~m}, 58 \mathrm{H}, \mathrm{PBu}_{3}\right.$ and $\left.\mathrm{C}_{8} \mathrm{H}_{17}\right), 0.82\left(\mathrm{~m}, 12 \mathrm{H}, \mathrm{C}_{8} \mathrm{H}_{17}\right), 0.56 \mathrm{ppm}$ (br, 8H, $\left.\mathrm{C}_{8} \mathrm{H}_{17}\right) .{ }^{31} \mathrm{P}$ NMR (161.9 MHz, $\left.\mathrm{CDCl}_{3}\right): \delta=3.85\left({ }^{1} J_{\mathrm{P}-\mathrm{Pt}}=2356 \mathrm{~Hz}, \mathrm{PBu}_{3}\right), 43.47 \mathrm{ppm}\left(\mathrm{PPh}_{3}\right)$. FAB-MS: $m / z 2390\left[\mathrm{M}^{+}\right]$. Anal. Calcd (\%) for $\mathrm{C}_{126} \mathrm{H}_{164} \mathrm{Au}_{2} \mathrm{P}_{4} \mathrm{Pt}$ : C, 63.28; H, 6.91. Found: C, 63.10; H, 7.02.

cis- $\left[\mathbf{P t}-\mathbf{T}\left(\mathbf{C}_{8}-\mathbf{F}\right) \mathbf{T}\right]_{\mathbf{n}}$ 


$$
\mathrm{Ph}_{2} \mathrm{P} \mathrm{Pt} \mathrm{PPh}_{2}
$$

$\left[\mathbf{H}-\mathbf{T}\left(\mathbf{C}_{\mathbf{8}} \mathbf{- F}\right) \mathbf{T}-\mathbf{H}_{1}(0.055 \mathrm{~g}, 0.126 \mathrm{mmol})\right.$ and $c i s-\left[\mathrm{PtCl}_{2}(\mathrm{dppe})\right](0.083 \mathrm{~g}, 0.126 \mathrm{mmol})$ were mixed in $\mathrm{NEt}_{3} / \mathrm{CH}_{2} \mathrm{Cl}_{2}(40 \mathrm{~mL}, 1: 10, \mathrm{v} / \mathrm{v})$ at room temperature in the presence of a small amount of $\mathrm{CuI}(3$ $\mathrm{mg})$. After the reaction mixture was stirred at ambient temperature overnight, it was filtered through a short silica gel pad to remove the catalyst and the insoluble solid. The filtrate was concentrated and poured into $\mathrm{MeOH}(50 \mathrm{~mL})$. The polymer powder was collected, washed with hexane and dried under vacuum $(0.097 \mathrm{~g}, 75 \%)$. IR $(\mathrm{KBr}): v=2108 \mathrm{~cm}^{-1}(\mathrm{C} \equiv \mathrm{C}) .{ }^{1} \mathrm{H}$ NMR $\left(400 \mathrm{MHz}, \mathrm{CDCl}_{3}\right): \delta=$ 8.09-7.99 (br, 8H, Ar), 7.52-7.28 (br, 16H, Ar), 6.99-6.84 (m, 2H, Ar), 2.45-2.47 (d, br, 4H, $\mathrm{PtPCH}_{2} \mathrm{CH}_{2} \mathrm{PPt}$ ), 1.80-1.75 (br, 4H, $\left.\mathrm{C}_{8} \mathrm{H}_{17}\right), 1.31-1.08$ (m, 20H, $\left.\mathrm{C}_{8} \mathrm{H}_{17}\right), 0.83-0.77$ (m, 6H, $\mathrm{C}_{8} \mathrm{H}_{17}$ ), 0.50 ppm (br, 4H, $\left.\mathrm{C}_{8} \mathrm{H}_{17}\right) .{ }^{31} \mathrm{P}$ NMR (161.9 MHz, $\left.\mathrm{CDCl}_{3}\right): \delta=41.80 \mathrm{ppm}\left({ }^{1} J_{\mathrm{P}-\mathrm{Pt}}=2282 \mathrm{~Hz}\right)$. Anal. Calcd (\%) for $\mathrm{C}_{59} \mathrm{H}_{64} \mathrm{P}_{2} \mathrm{Pt}: \mathrm{C}, 68.79 ; \mathrm{H}, 6.26$. Found: C, 68.55; H, 6.08.

$\left[\mathbf{P d}-\mathbf{T}\left(\mathrm{C}_{\mathbf{8}}-\mathbf{F}\right) \mathbf{T}\right]_{\mathbf{n}}$

$\left[\mathbf{H}-\mathbf{T}\left(\mathbf{C}_{\mathbf{8}} \mathbf{- F}\right) \mathbf{T}-\mathrm{H}_{1}(0.080 \mathrm{~g}, 0.183 \mathrm{mmol})\right.$ and trans-[PdCl $\left.2\left(\mathrm{PBu}_{3}\right)_{2}\right](0.106 \mathrm{~g}, 0.183 \mathrm{mmol})$ were 
added to a mixture of $\mathrm{NEt}_{3} / \mathrm{CH}_{2} \mathrm{Cl}_{2}(50 \mathrm{~mL}, 1: 1 \mathrm{v} / \mathrm{v})$ at room temperature. A small amount of CuI was added to the reaction mixture as the catalyst for the polymerization. After the reaction mixture was stirred at ambient temperature overnight, it was filtered through a short silica gel pad. The filtrate was concentrated and poured into $\mathrm{MeOH}(50 \mathrm{~mL})$. The polymer was precipitated out as a yellow-orange solid which was washed with hexane and dried under vacuum $(0.11 \mathrm{~g}, 65 \%)$. IR (KBr): $v(\mathrm{C} \equiv \mathrm{C}) 2107 \mathrm{~cm}^{-1} .{ }^{1} \mathrm{H}$ NMR (400 MHz, $\left.\mathrm{CDCl}_{3}\right): \delta=7.50$ (br, 4H, Ar), 7.25 (br, 2H, Ar), 2.12-0.60 ppm (m, 88H, $\mathrm{PBu}_{3}$ and $\left.\mathrm{C}_{8} \mathrm{H}_{17}\right) \cdot{ }^{13} \mathrm{C} \mathrm{NMR}\left(100.3 \mathrm{MHz}, \mathrm{CDCl}_{3}\right): \delta=151.25,141.26$,

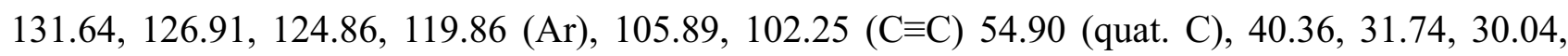
29.25, 23.72, 22.56, $14.05\left(\mathrm{C}_{8} \mathrm{H}_{17}\right), 26.63,25.19,24.36,13.81 \mathrm{ppm}\left(\mathrm{PBu}_{3}\right) .{ }^{31} \mathrm{P} \mathrm{NMR}(161.9 \mathrm{MHz}$, $\left.\mathrm{CDCl}_{3}\right): \delta=7.48$ ppm $\left(\mathrm{PBu}_{3}\right)$. Anal. Calcd (\%) for $\mathrm{C}_{57} \mathrm{H}_{94} \mathrm{P}_{2} \mathrm{Pd}: \mathrm{C}, 72.24 ; \mathrm{H}, 10.00$. Found: C, 71.99; H, 9.88 .

\section{$\left[T\left(C_{8}-F\right) T-P t-T\left(C_{8}-F\right) T\right]_{n}$}

The monomer $\left[\mathbf{T}\left(\mathbf{C}_{\mathbf{8}} \mathbf{- F}\right) \mathbf{T}-\mathbf{P t}-\mathbf{T}\left(\mathbf{C}_{\mathbf{8}} \mathbf{- F}\right) \mathbf{T}\right]_{\mathbf{1}}(0.10 \mathrm{~g}, 0.068 \mathrm{mmol})$ was added to pyridine $(8 \mathrm{~mL})$ and $\mathrm{CuCl}(1.1 \mathrm{mg}, 0.011 \mathrm{mmol})$ was added as the catalyst. The reaction mixture was stirred for $12 \mathrm{~h}$ at room temperature while a flow of oxygen was bubbled into it. After reaction, $\mathrm{CH}_{2} \mathrm{Cl}_{2}(30 \mathrm{~mL})$ was added to dissolve the precipitate formed and the solution was filtered through a short silica gel pad to remove the catalyst residue. The filtrate was concentrated. The residue was dissolved in a small 
amount of $\mathrm{CH}_{2} \mathrm{Cl}_{2}(6 \mathrm{~mL})$ and poured into $\mathrm{MeOH}(50 \mathrm{~mL})$. The precipitate was collected by filtration and washed with hexane. The product was obtained as a pale yellow crystalline solid. (0.095 g, 95\%). IR (KBr): v(C $\equiv \mathrm{C}) 2092 \mathrm{~cm}^{-1} .{ }^{1} \mathrm{H}$ NMR $\left(400 \mathrm{MHz}, \mathrm{CDCl}_{3}\right): \delta=7.59-7.42(\mathrm{~m}, 8 \mathrm{H}$, Ar), 7.26-7.25 (br, 4H, Ar), 2.20 (br, 12H, $\mathrm{PBu}_{3}$ ), 1.89 (br, 8H, $\left.\mathrm{C}_{8} \mathrm{H}_{17}\right), 1.67$ (br, $\left.12 \mathrm{H}, \mathrm{PBu}_{3}\right), 1.49$ (m, 12H, $\left.\mathrm{PBu}_{3}\right), 1.23-0.94\left(\mathrm{~m}, 58 \mathrm{H}, \mathrm{C}_{8} \mathrm{H}_{17}\right.$ and $\left.\mathrm{PBu}_{3}\right), 0.86-0.81\left(\mathrm{~m}, 12 \mathrm{H}, \mathrm{C}_{8} \mathrm{H}_{17}\right), 0.60 \mathrm{ppm}(\mathrm{br}, 8 \mathrm{H}$, $\left.\mathrm{C}_{8} \mathrm{H}_{17}\right) .{ }^{13} \mathrm{C} \mathrm{NMR}\left(100.3 \mathrm{MHz}, \mathrm{CDCl}_{3}\right): \delta=150.74,142.42,137.16,131.21,129.49,128.35,126.47$, 124.97, 119.91, 119.46, 119.33, 119.15 (Ar), 110.25, 84.95, 83.28, 74.06 (C $\equiv \mathrm{C}) 54.83$ (quat. C), 40.48, 31.78, 30.12, 29.31, 23.74, 22.59, $14.19\left(\mathrm{C}_{8} \mathrm{H}_{17}\right), 26.37,24.46,23.94,13.97 \mathrm{ppm}\left(\mathrm{PBu}_{3}\right) .{ }^{31} \mathrm{P}$ NMR (161.9 MHz, $\left.\mathrm{CDCl}_{3}\right): \delta=3.96 \mathrm{ppm}\left({ }^{1} J_{\mathrm{P}-\mathrm{Pt}}=2350 \mathrm{~Hz}\right)$. Anal. Calcd $(\%)$ for $\mathrm{C}_{90} \mathrm{H}_{134} \mathrm{P}_{2} \mathrm{Pt}: \mathrm{C}$, 73.38; H, 9.17. Found: C, 73.20; H, 9.25.

$\left[P d-T\left(C_{8}-F\right) T-P t-T\left(C_{8}-F\right) T\right]_{n}$

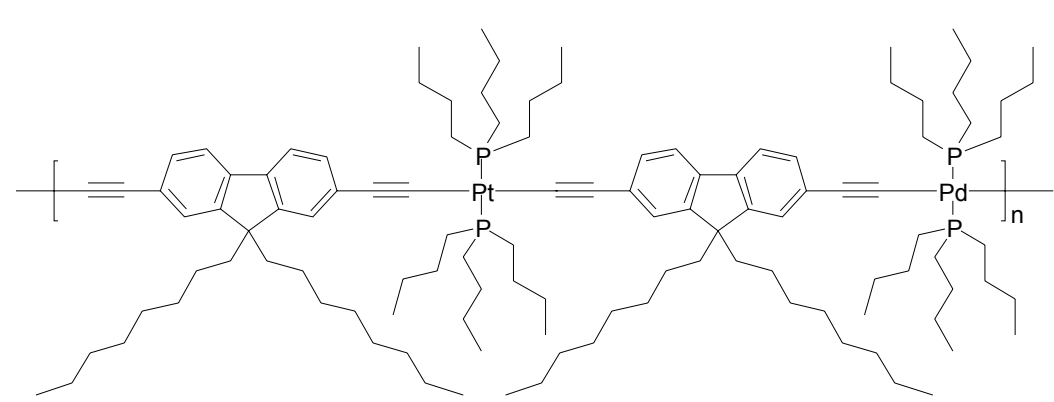

$\left[\mathbf{T}\left(\mathbf{C}_{\mathbf{8}} \mathbf{- F}\right) \mathbf{T}-\mathbf{P t}-\mathbf{T}\left(\mathbf{C}_{\mathbf{8}} \mathbf{- F}\right) \mathbf{T}\right]_{\mathbf{1}}(0.15 \mathrm{~g}, 0.102 \mathrm{mmol})$ and trans-[PdCl$\left.\left(\mathrm{PBu}_{3}\right)_{2}\right](0.059 \mathrm{~g}, 0.102 \mathrm{mmol})$ were added to a mixture of $\mathrm{NEt}_{3} / \mathrm{CH}_{2} \mathrm{Cl}_{2}(40 \mathrm{~mL}, 1: 1 \mathrm{v} / \mathrm{v})$ at room temperature. A small amount of $\mathrm{CuI}$ was added to the reaction mixture as the catalyst. After the reaction mixture was stirred at ambient temperature overnight, it was filtered through a short pad of silica gel and the solvent was removed in vacuo. The residue was dissolved in a small amount of $\mathrm{CH}_{2} \mathrm{Cl}_{2}(6 \mathrm{~mL})$ and poured into $\mathrm{MeOH}(50 \mathrm{~mL})$. The precipitate was collected by filtration and washed with hexane. The product 
was obtained as a yellow-orange solid $(0.171 \mathrm{~g}, 85 \%)$. IR (KBr): $v(\mathrm{C} \equiv \mathrm{C}) 2095 \mathrm{~cm}^{-1}$. ${ }^{1} \mathrm{H}$ NMR (400 $\left.\mathrm{MHz}, \mathrm{CDCl}_{3}\right): \delta=7.49-7.45(\mathrm{~m}, 4 \mathrm{H}, \mathrm{Ar}), 7.25-7.22(\mathrm{~m}, 8 \mathrm{H}, \mathrm{Ar}), 2.22-2.07\left(\mathrm{~m}, 24 \mathrm{H}, \mathrm{PtPBu}_{3}\right.$ and $\left.\mathrm{PdPBu}_{3}\right), 1.86\left(\mathrm{br}, 8 \mathrm{H}, \mathrm{C}_{8} \mathrm{H}_{17}\right), 1.67-1.41\left(\mathrm{~m}, 48 \mathrm{H}, \mathrm{PtPBu}_{3}\right.$ and $\left.\mathrm{PdPBu}_{3}\right), 1.25-0.86\left(\mathrm{~m}, 76 \mathrm{H}, \mathrm{C}_{8} \mathrm{H}_{17}\right.$, $\mathrm{PtPBu}_{3}$ and $\left.\mathrm{PdPBu}_{3}\right), 0.85-0.81\left(\mathrm{~m}, 12 \mathrm{H}, \mathrm{C}_{8} \mathrm{H}_{17}\right), 0.62 \mathrm{ppm}\left(\mathrm{br}, 8 \mathrm{H}, \mathrm{C}_{8} \mathrm{H}_{17}\right) .{ }^{13} \mathrm{C} \mathrm{NMR}(100.3 \mathrm{MHz}$, $\left.\mathrm{CDCl}_{3}\right): \delta=150.29,142.43,138.41,131.46,129.51,126.73,125.14,124.78,119.68,119.30,118.93$, 118.79, (Ar), 111.66, 110.26, 106.07, 100.50 (C $\equiv \mathrm{C}) 54.50$ (quat. C), 40.72, 31.78, 30.26, 29.40, 23.79, 22.58, $14.58\left(\mathrm{C}_{8} \mathrm{H}_{17}\right), 26.67,25.20,24.81,13.85\left(\mathrm{PdPBu}_{3}\right), 26.37,24.45,23.95,13.82 \mathrm{ppm}$ $\left(\mathrm{PtPBu}_{3}\right) .{ }^{31} \mathrm{P}$ NMR $\left(161.9 \mathrm{MHz}, \mathrm{CDCl}_{3}\right): \delta=3.85\left({ }^{1} J_{\mathrm{P}-\mathrm{Pt}}=2362 \mathrm{~Hz}\right)\left(\mathrm{PtPBu}_{3}\right), 7.42 \mathrm{ppm}\left(\mathrm{PdPBu}_{3}\right)$. Anal. Calcd (\%) for $\mathrm{C}_{114} \mathrm{H}_{188} \mathrm{P}_{4} \mathrm{PdPt}$ : C, 69.01; H, 9.55. Found: C, 68.78; H, 9.64.

\section{$\left[\mathrm{Pd}-\mathrm{T}\left(\mathrm{C}_{\mathbf{8}}-\mathrm{F}\right) \mathrm{T}-\mathrm{Hg}-\mathrm{T}\left(\mathrm{C}_{\mathbf{8}}-\mathrm{F}\right) \mathrm{T}\right]_{\mathrm{n}}$}

$\left[\mathbf{T}\left(\mathbf{C}_{\mathbf{8}}-\mathbf{F}\right) \mathbf{T}-\mathbf{H g}-\mathbf{T}\left(\mathbf{C}_{\mathbf{8}} \mathbf{- F}\right) \mathbf{T}\right]_{1}(0.15 \mathrm{~g}, 0.140 \mathrm{mmol})$ and trans-[ $\left.\mathrm{PdCl}_{2}\left(\mathrm{PBu}_{3}\right)_{2}\right](0.081 \mathrm{~g}, 0.140 \mathrm{mmol})$ were added to a mixture of $\mathrm{NEt}_{3} / \mathrm{CH}_{2} \mathrm{Cl}_{2}(40 \mathrm{~mL}, 1: 1 \mathrm{v} / \mathrm{v})$ at room temperature. A small amount of $\mathrm{CuI}$ was added to the reaction mixture as the catalyst. After the reaction mixture was stirred at ambient temperature overnight, it was filtered through a short pad of silica gel to remove the catalyst. The solvent was removed under reduced pressure. The residue was dissolved in a small amount of $\mathrm{CH}_{2} \mathrm{Cl}_{2}(6 \mathrm{~mL})$ and poured into $\mathrm{MeOH}(50 \mathrm{~mL})$. The precipitate was collected by filtration and washed with hexane. The product was obtained as a yellow solid $(0.175 \mathrm{~g}, 80 \%)$. IR $(\mathrm{KBr}): v(\mathrm{C} \equiv \mathrm{C})$ $2090 \mathrm{~cm}^{-1} .{ }^{1} \mathrm{H}$ NMR (400 MHz, $\left.\mathrm{CDCl}_{3}\right): \delta=7.52-7.48(\mathrm{~m}, 8 \mathrm{H}, \mathrm{Ar}), 7.27-7.22(\mathrm{~m}, 4 \mathrm{H}, \mathrm{Ar}), 2.11(\mathrm{~m}$, 
12H, $\left.\mathrm{PBu}_{3}\right), 1.96\left(\mathrm{br}, 8 \mathrm{H}, \mathrm{C}_{8} \mathrm{H}_{17}\right), 1.67$ (br, 12H, $\left.\mathrm{PBu}_{3}\right), 1.46\left(\mathrm{~m}, 12 \mathrm{H}, \mathrm{PBu}_{3}\right), 1.21-0.92(\mathrm{~m}, 58 \mathrm{H}$, $\mathrm{C}_{8} \mathrm{H}_{17}$ and $\left.\mathrm{PBu}_{3}\right), 0.84\left(\mathrm{~m}, 12 \mathrm{H}, \mathrm{C}_{8} \mathrm{H}_{17}\right), 0.62 \mathrm{ppm}\left(\mathrm{br}, 8 \mathrm{H}, \mathrm{C}_{8} \mathrm{H}_{17}\right) .{ }^{13} \mathrm{C} \mathrm{NMR}\left(100.3 \mathrm{MHz}, \mathrm{CDCl}_{3}\right): \delta$ $=151.26,150.80,141.17,131.58,129.71,126.92,124.78,120.85,120.23,119.70,119.50,118.95$ (Ar), 109.40, 105.90, 95.28, 83.53 (C $\equiv C) 55.25$ (quat. C), 40.38, 31.77, 29.93, 29.29, 23.74, 22.59, $14.08\left(\mathrm{C}_{8} \mathrm{H}_{17}\right), 26.66,25.22,24.38,13.83 \mathrm{ppm}\left(\mathrm{PBu}_{3}\right) .{ }^{31} \mathrm{P} \mathrm{NMR}\left(161.9 \mathrm{MHz}, \mathrm{CDCl}_{3}\right): \delta=7.53 \mathrm{ppm}$ $\left(\mathrm{PBu}_{3}\right)$. Anal. Calcd (\%) for $\mathrm{C}_{90} \mathrm{H}_{134} \mathrm{HgP}_{2} \mathrm{Pd}: \mathrm{C}, 68.20 ; \mathrm{H}, 8.52$. Found: $\mathrm{C}, 68.01 ; \mathrm{H}, 8.33$.

\section{$\left[\mathrm{Hg}-\mathrm{T}\left(\mathrm{C}_{8}-\mathrm{F}\right) \mathrm{T}-\mathrm{Pt}-\mathrm{T}\left(\mathrm{C}_{8}-\mathrm{F}\right) \mathrm{T}\right]_{\mathrm{n}}$}

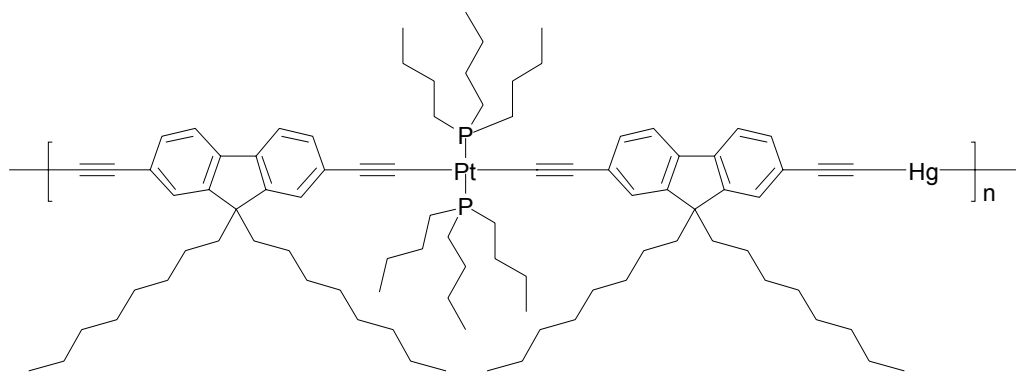

[T( $\mathbf{C}_{\mathbf{8}}$-F)T-Pt-T(C $\mathbf{C}_{\mathbf{8}}$-F)T $]_{\mathbf{1}}(0.150 \mathrm{~g}, 0.102 \mathrm{mmol})$ and $\mathrm{HgCl}_{2}(0.028 \mathrm{~g}, 0.102 \mathrm{mmol})$ were dissolved in $\mathrm{MeOH} / \mathrm{CH}_{2} \mathrm{Cl}_{2}(40 \mathrm{~mL}, 1: 50 \mathrm{v} / \mathrm{v})$ at room temperature. A basic $\mathrm{NaOMe}$ solution $(1.2 \mathrm{~mL}, 0.2$ $\mathrm{mmol} / \mathrm{L}$ in $\mathrm{MeOH}$ ) was added. After the reaction mixture was stirred at ambient temperature overnight, it was filtered through a short pad of silica gel to remove the insoluble solid. The solvent was removed under reduced pressure. The residue was dissolved in a small amount of $\mathrm{CH}_{2} \mathrm{Cl}_{2}(6 \mathrm{~mL})$ and poured into $\mathrm{MeOH}(50 \mathrm{~mL})$. The precipitate was collected by filtration and washed with hexane. The product was obtained as an off-white solid. $(0.125 \mathrm{~g}, 73 \%)$. IR $(\mathrm{KBr}): v(\mathrm{C} \equiv \mathrm{C}) 2093 \mathrm{~cm}^{-1} .{ }^{1} \mathrm{H}$ NMR (400 MHz, $\left.\mathrm{CDCl}_{3}\right): \delta=7.56-7.44(\mathrm{~m}, 8 \mathrm{H}, \mathrm{Ar}), 7.27-7.24(\mathrm{~m}, 4 \mathrm{H}, \mathrm{Ar}), 2.12$ (br, 12H, Bu), 1.90 (br, 8H, $\left.\mathrm{C}_{8} \mathrm{H}_{17}\right), 1.66$ (br, $\left.12 \mathrm{H}, \mathrm{Bu}\right), 1.49-1.47(\mathrm{~m}, 12 \mathrm{H}, \mathrm{Bu}), 1.21-0.93\left(\mathrm{~m}, 58 \mathrm{H}, \mathrm{C}_{8} \mathrm{H}_{17}\right.$ and $\mathrm{Bu}), 0.83\left(\mathrm{~m}, 12 \mathrm{H}, \mathrm{C}_{8} \mathrm{H}_{17}\right), 0.60 \mathrm{ppm}\left(\mathrm{br}, 8 \mathrm{H}, \mathrm{C}_{8} \mathrm{H}_{17}\right) .{ }^{31} \mathrm{P} \mathrm{NMR}\left(161.9 \mathrm{MHz}, \mathrm{CDCl}_{3}\right): \delta=3.96 \mathrm{ppm}$ 
$\left({ }^{1} J_{\mathrm{P}-\mathrm{Pt}}=2352 \mathrm{~Hz}\right)$. Anal. Calcd (\%) for $\mathrm{C}_{90} \mathrm{H}_{134} \mathrm{HgP}_{2} \mathrm{Pt}$ : C, 64.59; H, 8.07. Found: C, 64.45; H, 7.76.

\section{$\left[T\left(C_{8}-F\right) T-H g-T\left(C_{8}-F\right) T\right]_{n}$}

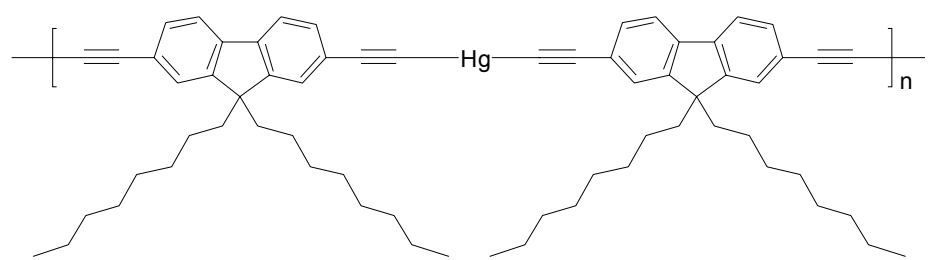

The monomer $\left[\mathbf{T}\left(\mathbf{C}_{\mathbf{8}}-\mathbf{F}\right) \mathbf{T}-\mathbf{H g}-\mathbf{T}\left(\mathbf{C}_{\mathbf{8}} \mathbf{- F}\right) \mathbf{T}\right]_{1}(0.10 \mathrm{~g}, 0.093 \mathrm{mmol})$ was added to pyridine $(8 \mathrm{~mL})$ and $\mathrm{CuCl}(1.5 \mathrm{mg} 0.015 \mathrm{mmol})$ was added as the catalyst. The reaction mixture was stirred for $12 \mathrm{~h}$ at ambient temperature while a flow of oxygen was bubbled through it. After the reaction, $\mathrm{CH}_{2} \mathrm{Cl}_{2}(30$ $\mathrm{mL}$ ) was added and the reaction mixture was filtered through a short silica gel pad to remove the catalyst residue. The filtrate was then concentrated. The residue was dissolved in a small amount of $\mathrm{CH}_{2} \mathrm{Cl}_{2}(6 \mathrm{~mL})$ and poured into $\mathrm{MeOH}(50 \mathrm{~mL})$. The precipitate was collected by filtration and washed with hexane. The product was obtained as a pale yellow solid. (0.090 g, 90\%). IR (KBr): $v(\mathrm{C} \equiv \mathrm{C}) 2141 \mathrm{~cm}^{-1} .{ }^{1} \mathrm{H}$ NMR $\left(400 \mathrm{MHz}, \mathrm{CDCl}_{3}\right): \delta=7.66-7.63(\mathrm{~m}, 4 \mathrm{H}, \mathrm{Ar}), 7.51-7.48$ (m, 8H, Ar), 1.91 (br, $\left.8 \mathrm{H}, \mathrm{C}_{8} \mathrm{H}_{17}\right), 1.21-1.03\left(\mathrm{~m}, 40 \mathrm{H}, \mathrm{C}_{8} \mathrm{H}_{17}\right), 0.82-0.81\left(\mathrm{~m}, 12 \mathrm{H}, \mathrm{C}_{8} \mathrm{H}_{17}\right), 0.57$ ppm (br, 8H, $\left.\mathrm{C}_{8} \mathrm{H}_{17}\right) .{ }^{13} \mathrm{C} \mathrm{NMR}\left(100.3 \mathrm{MHz}, \mathrm{CDCl}_{3}\right): \delta=151.25,151.14,140.95,131.51,131.24,126.92,126.72$, 121.00, 120.81, 120.54, 120.21, 120.06 (Ar), 106.85, 84.50, 83.09, 74.49 (C $\equiv C) 55.22$ (quat. C), 40.22, 31.76, 29.95, 29.22, 23.70, 22.58, 14.09 ppm $\left(\mathrm{C}_{8} \mathrm{H}_{17}\right)$. Anal. Calcd (\%) for $\mathrm{C}_{66} \mathrm{H}_{80} \mathrm{Hg}$ : C, 73.81; H, 7.51. Found: C, 73.55; H, 7.70. 
X-ray Crystallographic Details. Good-quality crystals of the samples were grown at room temperature by slow evaporation of their solutions in a $\mathrm{CH}_{2} \mathrm{Cl}_{2} /$ hexane mixture. Geometric and intensity data were collected at $293 \mathrm{~K}$ using graphite-monochromated Mo-K $\alpha$ radiation $(\lambda=$ $0.71073 \AA$ ) on a Bruker Axs SMART 1000 CCD diffractometer. The collected frames were processed with the software $\mathrm{SAINT}^{3}$ and an absorption correction (SADABS) ${ }^{[4]}$ was applied to the collected reflections. The structure was solved by the Direct methods (SHELXTL) ${ }^{[5]}$ in conjunction with standard difference Fourier techniques and subsequently refined by full-matrix least-squares analyses on $F^{2}$. Hydrogen atoms were generated in their idealized positions and all non-hydrogen atoms were assigned with anisotropic displacement parameters.

Theoretical Computations. Density functional calculations at the B3LYP level were performed based on their experimental geometries from the $\mathrm{X}$-ray data. The basis set used for $\mathrm{C}$ and $\mathrm{H}$ atoms was 6-31G while effective core potentials with a LanL2DZ basis set were employed for $\mathrm{P}, \mathrm{Hg}, \mathrm{Pd}$ and $\mathrm{Pt}$ atoms. Polarization functions were added for phosphorus $\left(\zeta_{\mathrm{d}}(\mathrm{P})=0.34\right) .{ }^{[6]}$ All the calculations were carried out using the Gaussian 03 program. ${ }^{[7]}$ Mulliken population analyses were done with MullPop. ${ }^{[8]}$

References:

[1] a) M. S. Khan, M. R. A. Al-Mandhary, M. K. Al-Suti, B. Ahrens, M. F. Mahon, L. Male, P. R. Raithby, C. E. Boothby, A. Köhler, Dalton Trans. 2003, 74. b) S. Maruyama, X.-T. Tao, H. Hokari, T. Noh, Y. Zhang, T. Wada, H. Sasabe, H. Suzuki, T. Watanabe, S. Miyata, J. Mater. Chem. 1999, 9, 893. 
[2] W.-Y. Wong, L. Liu, J.-X. Shi, Angew. Chem. Int. Ed. 2003, 42, 4064.

[3] SAINT+, ver. 6.02a, Bruker Analytical X-ray System, Inc., Madison, WI, 1998.

[4] G. M. Sheldrick, SADABS, Empirical Absorption Correction Program; University of Göttingen, Germany, 1997.

[5] G. M. Sheldrick, SHELXTL ${ }^{\mathrm{TM}}$, Reference manual, ver. 5.1, Madison, WI, 1997.

[6] a) W. R. Wadt, P. J. Hay, J. Chem. Phys. 1985, 82, 284. b) P. J. Hay, W. R. Wadt, J. Chem. Phys. 1985, 82, 299.

[7] M. J. Frisch, G. W. Trucks, H. B. Schlegel, G. E. Scuseria, M. A. Robb, J. R. Cheeseman, J. A. Montgomery, T. Jr. Vreven, K. N. Kudin, J. C. Burant, J. M. Millam, S. S. Iyengar, J. Tomasi, V. Barone, B. Mennucci, M. Cossi, G. Scalmani, N. Rega, G. A. Petersson, H. Nakatsuji, M. Hada, M. Ehara, K. Toyota, R. Fukuda, J. Hasegawa, M. Ishida, T. Nakajima, Y. Honda, O. Kitao, H. Nakai, M. Klene, X. Li, J. E. Knox, H. P. Hratchian, J. B. Cross, C. Adamo, J. Jaramillo, R. Gomperts, R. E. Stratmann, O. Yazyev, A. J. Austin, R. Cammi, C. Pomelli, J. W. Ochterski, P. Y. Ayala, K. Morokuma, G. A. Voth, P. Salvador, J. J. Dannenberg, V. G. Zakrzewski, S. Dapprich, A. D. Daniels, M. C. Strain, O. Farkas, D. K. Malick, A. D. Rabuck, K. Raghavachari, J. B. Foresman, J. V. Ortiz, Q. Cui, A. G. Baboul, S. Clifford, J. Cioslowski, B. B. Stefanov, G. Liu, A. Liashenko, P. Piskorz, I. Komaromi, R. L. Martin, D. J. Fox, T. Keith, M. A. Al-Laham, C. Y. Peng, A. Nanayakkara, M. Challacombe, P. M. W. Gill, B. Johnson, W. Chen, M. W. Wong, C. Gonzalez, J. A. Pople, Gaussian 03, revision B05, Gaussian, Inc., Pittsburgh, PA, 2003.

[8] MullPop, a program written by Reinaldo Pis Diez at the National University of La Plata, Argentina. 
Optical-Limiting Measurements. To study the optical-limiting properties, the Z-scan measurements were performed with a Q-switched Nd:YAG laser at the repetition rate of $10 \mathrm{~Hz}$. The arrangement of the system is shown in Figure S1. The laser was frequency doubled with an output wavelength of $532 \mathrm{~nm}$ with $10 \mathrm{~ns}$ pulse width for Gaussian mode by a frequency double crystal (FDC). The laser beam was then split into two beams by a beam splitter (BS). One was used as the reference beam, which was received by a detector $\left(D_{1}\right)$, the other was for the sample measurement and it was focused with a lens $\left(\mathrm{L}_{1}, f=20 \mathrm{~cm}\right)$. After transmitting through the sample $(\mathrm{S})$, the light beam entered another detector $\left(D_{2}\right)$. The sample to be measured was moved along a rail to change the incident irradiance on it. The incident and transmitted energies were detected simultaneously by the two detectors $\mathrm{D}_{1}$ and $\mathrm{D}_{2}$ (LPE-1A) individually. The performance of each of our samples was measured as an $92 \%$ transmitting solution at $532 \mathrm{~nm}$ in $\mathrm{CH}_{2} \mathrm{Cl}_{2}$ in a $1 \mathrm{~mm}$ quartz cell. Experiments with $\mathrm{CH}_{2} \mathrm{Cl}_{2}$ alone afforded no detectable optical-limiting effect, indicating that the solvent contribution is negligible.

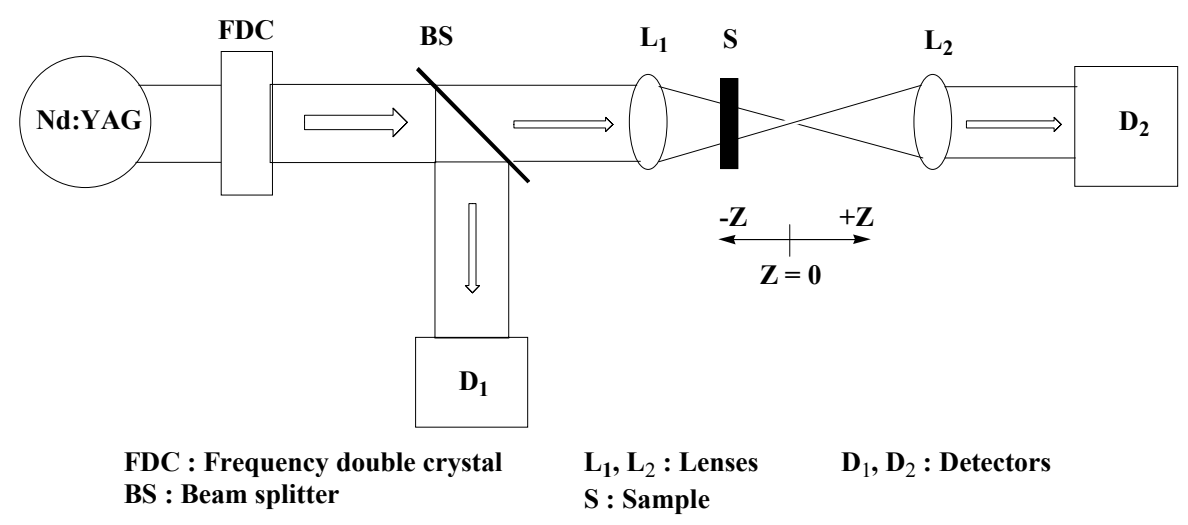

Figure S1. Experimental set-up for the measurement of optical-limiting properties. 
Mathematical treatments governing the RSA process. It can be assumed that the molecular energy levels of the metallaynes could be reduced to a three-level system with no saturation, diffusion, or recombination during the pulse. Thus we neglected all excitations higher than the first singlet excitation and assumed that the intersystem crossing rate was fast (compared with the pulse duration) and that all of the initially excited charges are in the lowest triplet state. Excitation of the first triplet state then leads to excited-state absorption. The equations governing the absorption are

$$
\begin{aligned}
& \frac{d I}{d z}=-\alpha I-\sigma_{e x} N(t) I \\
& \frac{d N}{d z}=\frac{\alpha I}{\hbar \omega}
\end{aligned}
$$

where $I$ is the input laser pulse intensity, $\alpha$ is the linear absorption coefficient, $\sigma_{\mathrm{ex}}$ is the excited-state cross section, $N$ is the number density of molecules in the excited state, and $\omega$ is the angular frequency of the laser. Equations (1) and (2) can be combined to get (3):

$$
\frac{d I}{d z}=-\alpha I-\frac{\sigma_{e x} \alpha I}{\hbar \omega} \int_{-\infty}^{t} I\left(t^{\prime}\right) d t^{\prime}
$$

Solving the equation for the fluence and integrating over the spatial extent of the laser pulse, the normalized energy transmission $T$ for RSA can be obtained as

$$
\begin{gathered}
T=\ln \left(1+\frac{q_{0}}{1+x^{2}}\right) /\left(\frac{q_{0}}{1+x^{2}}\right) \\
q_{0}=\frac{\sigma_{e x} F_{0}(r=0) \alpha L_{e f f}}{2 \hbar \omega}
\end{gathered}
$$

where $x=\mathrm{Z} / \mathrm{Z}_{0}, \mathrm{Z}$ is the distance of the sample from the focus, $L_{\mathrm{eff}}=[1-\exp (-\alpha L)] / \alpha$, and $F_{0}(r=$ $0)$ is the on-axis fluence at the focus. 


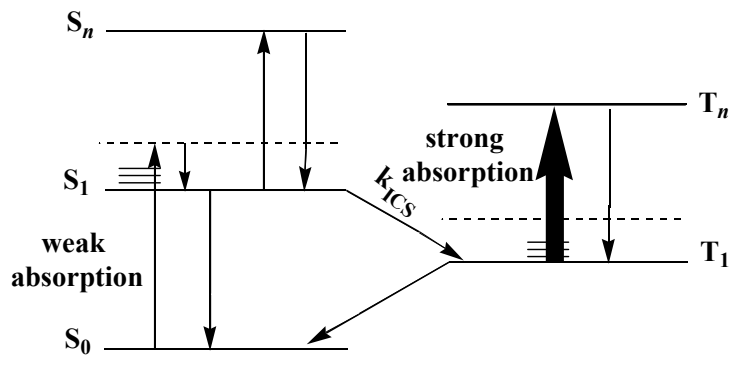

(a)

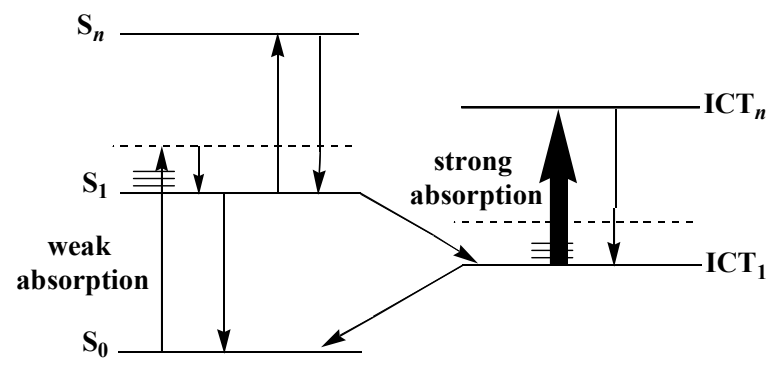

(b)

Figure S2. Energy level diagrams for the five-leveled reverse saturable absorption (RSA) optical-limiting mechanisms.

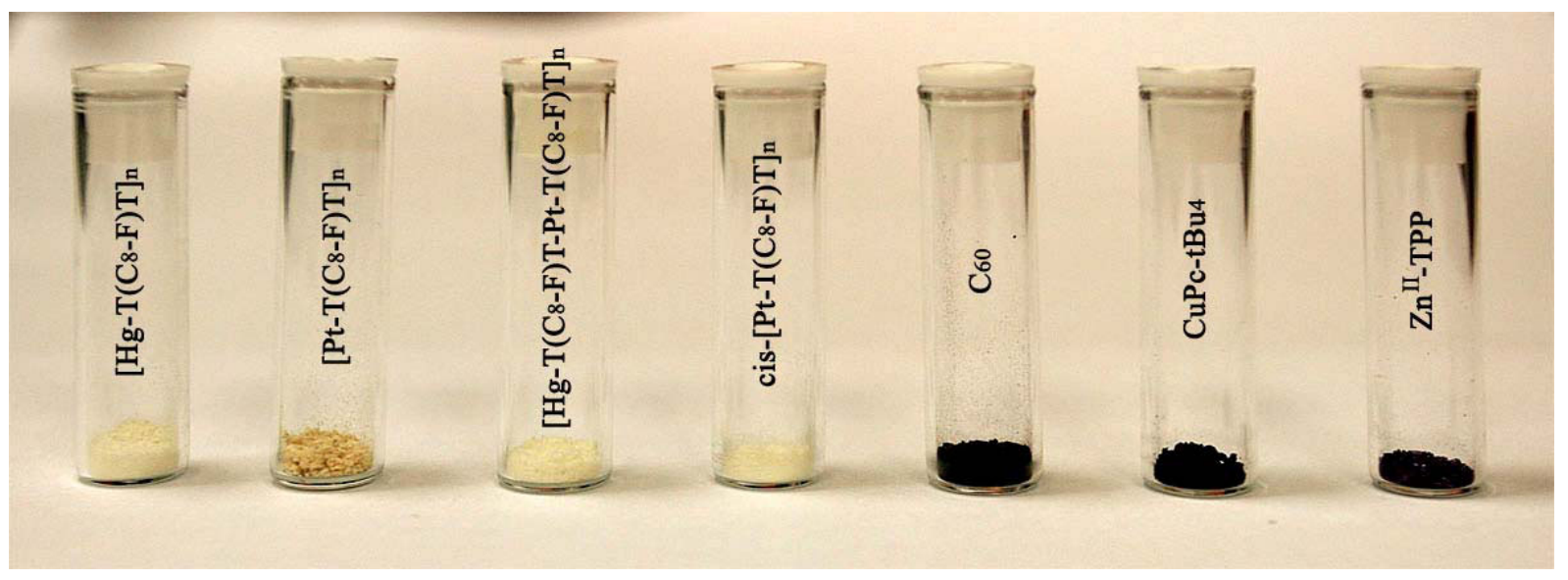

Figure S3. The sample colors of different materials in the solid state. 

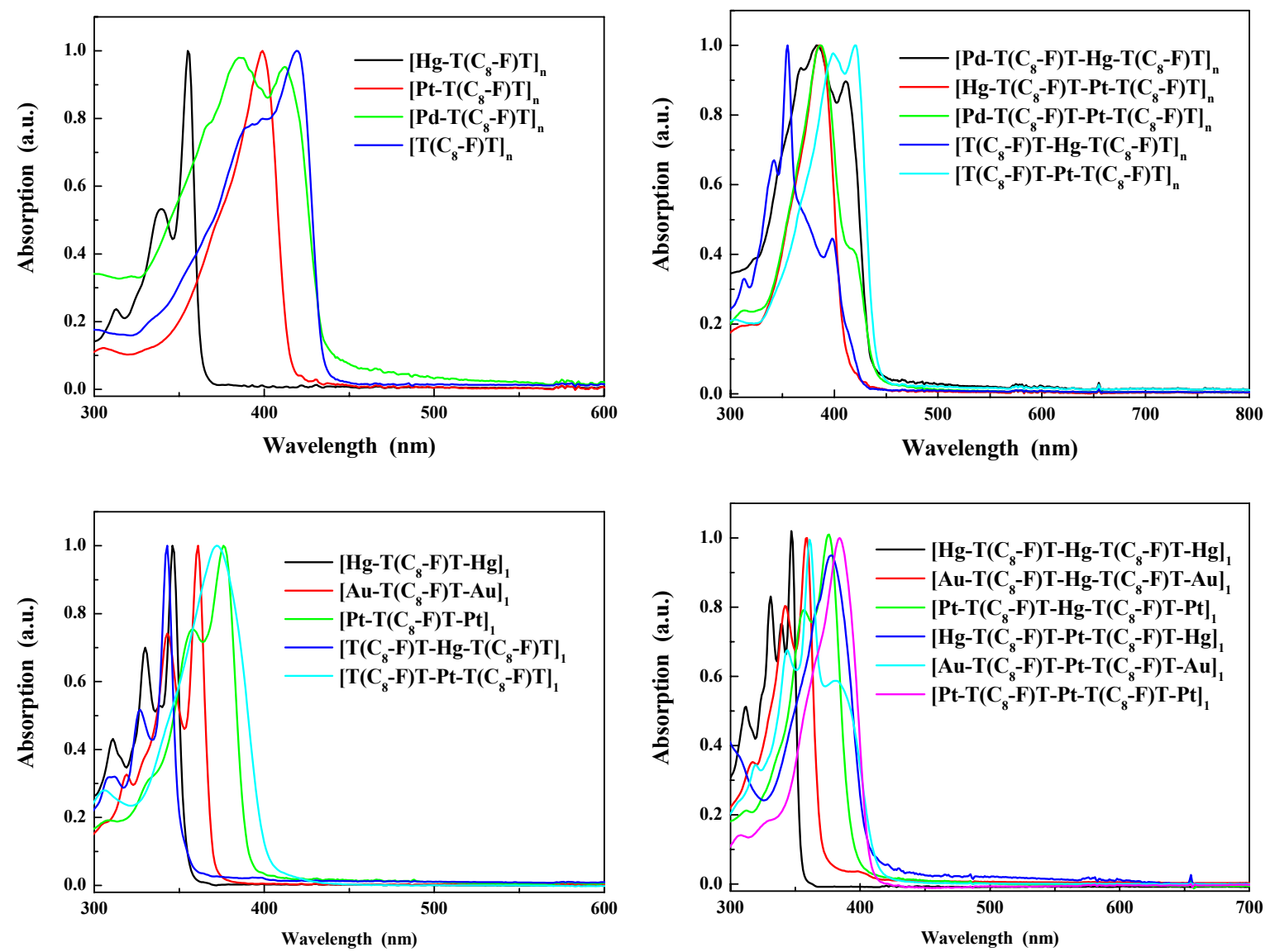

Figure S4. Linear absorption spectra of (poly)metallaynes at r.t. in $\mathrm{CH}_{2} \mathrm{Cl}_{2}$. 

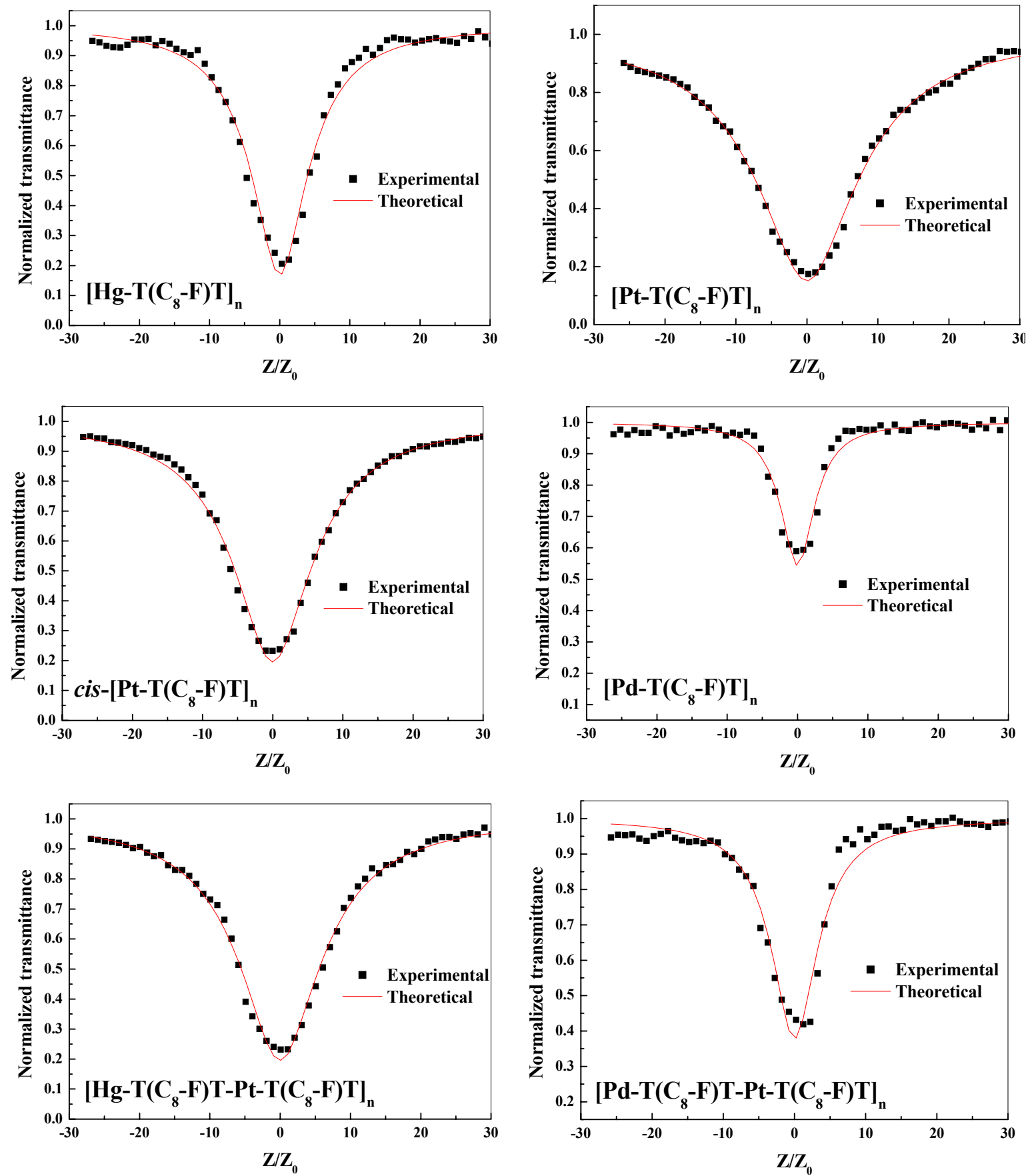

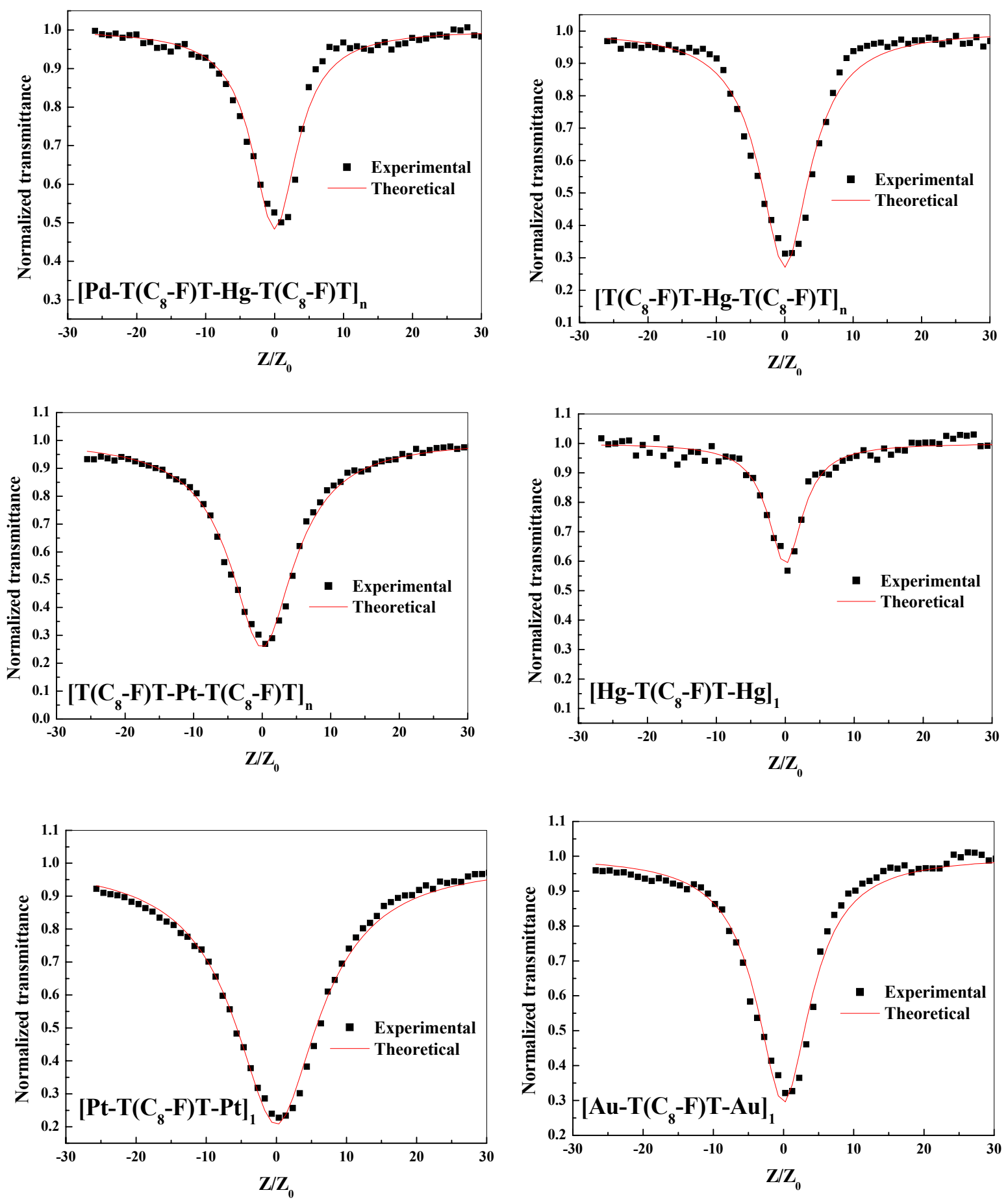

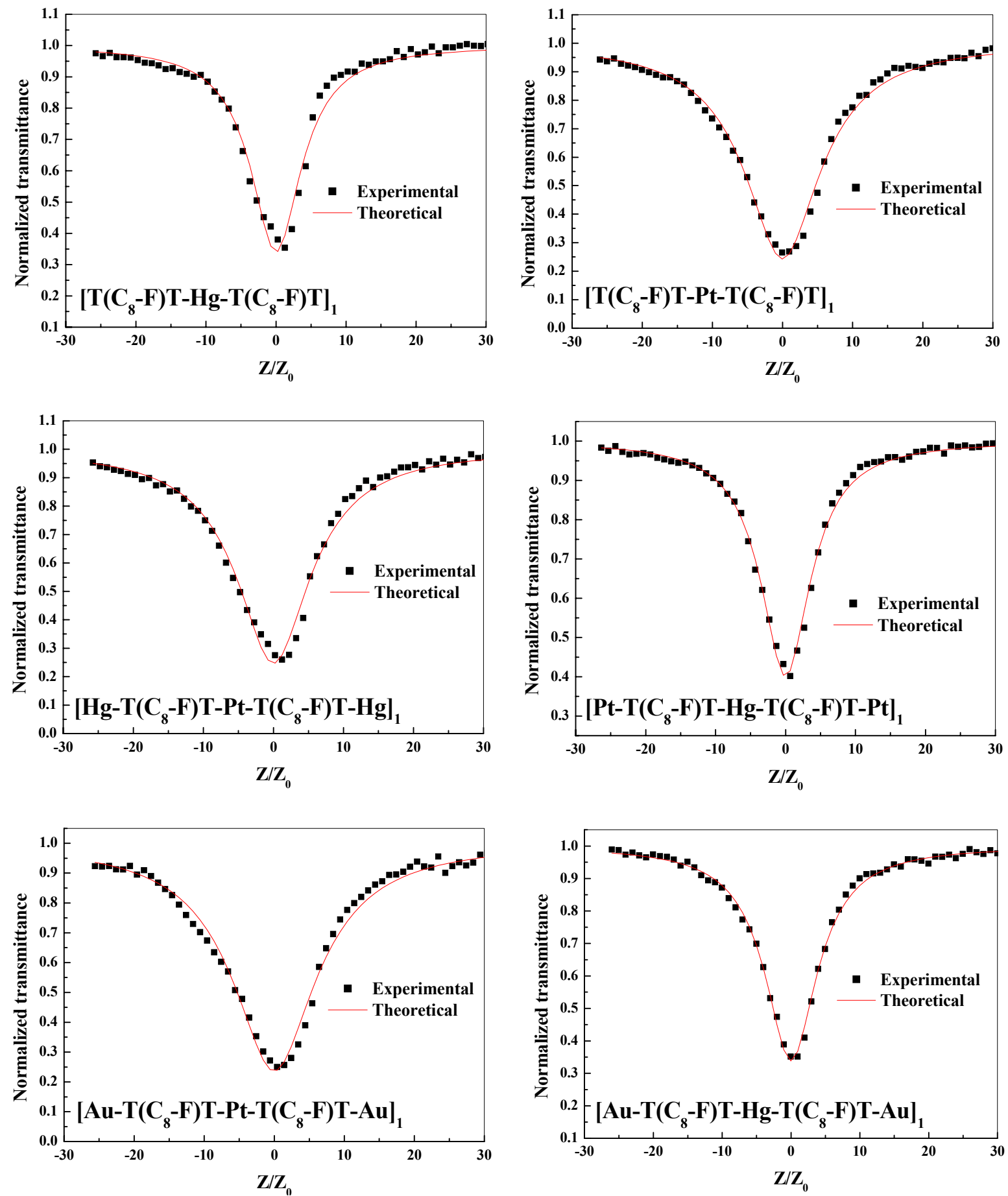

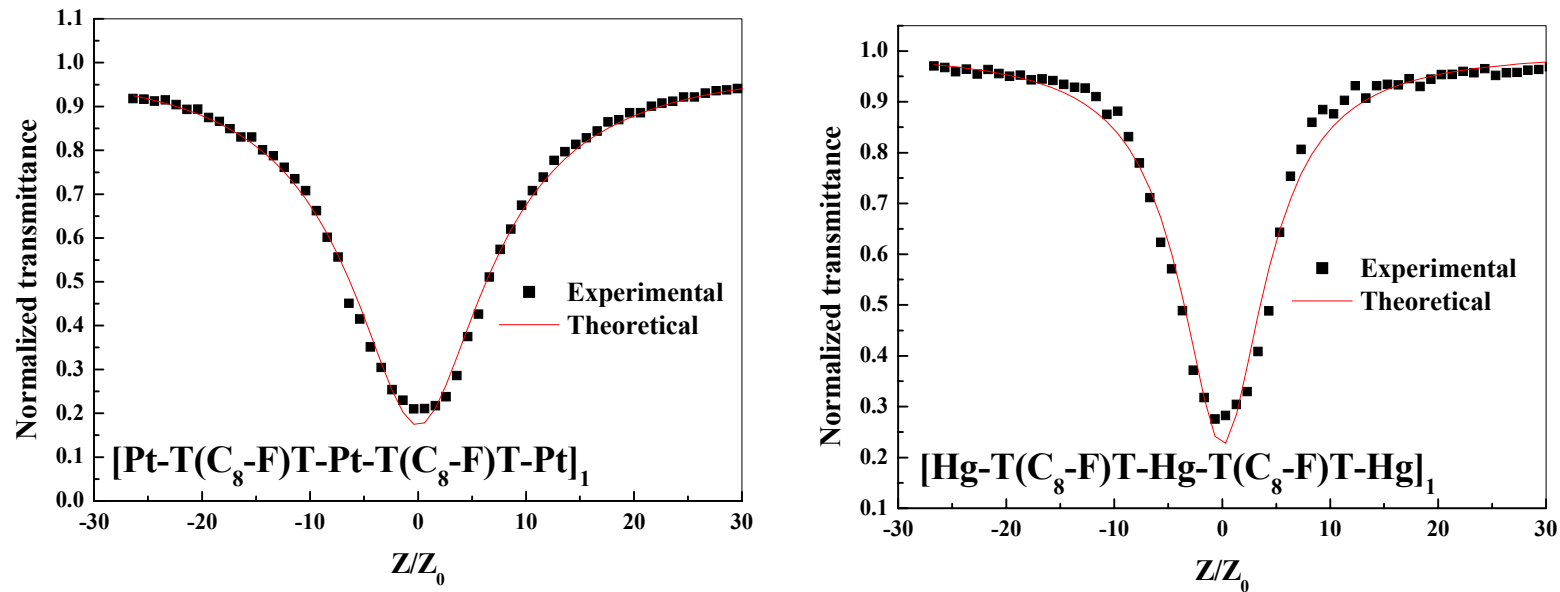

Figure S5. The Z-scan curves and their RSA numerical fits for (poly)metallaynes. 


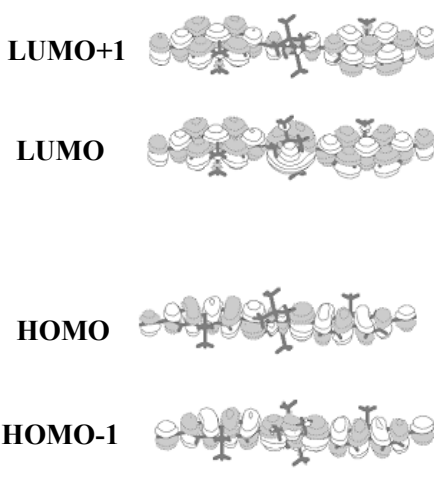

(a)
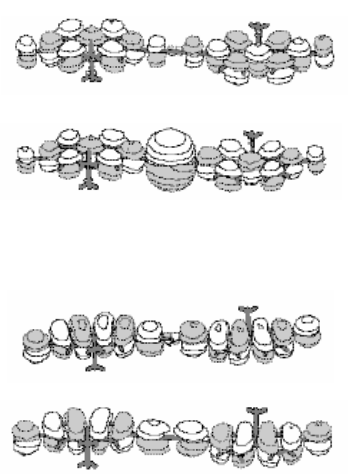

(b)
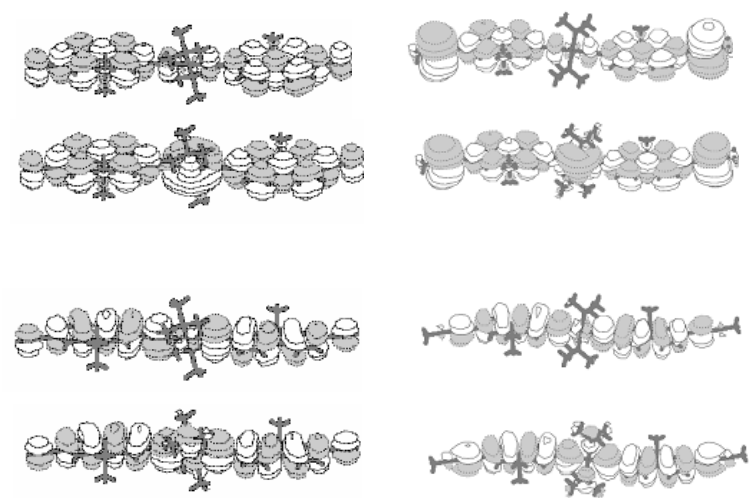

(c)

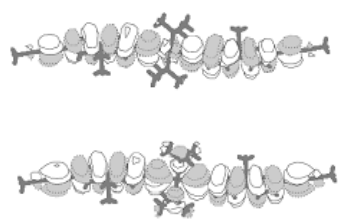

(d)

Figure S6. Spatial plots of the HOMOs and LUMOs for the model compounds of the organometallic polyynes.

(a) $\left[\mathrm{T}\left(\mathrm{C}_{8}-\mathrm{F}\right) \mathrm{T}-\mathrm{Pt}-\mathrm{T}\left(\mathrm{C}_{8}-\mathrm{F}\right) \mathrm{T}\right]_{1}$

(b) $\left[\mathrm{T}\left(\mathrm{C}_{8}-\mathrm{F}\right) \mathrm{T}-\mathrm{Hg}-\mathrm{T}\left(\mathrm{C}_{8}-\mathrm{F}\right) \mathrm{T}\right]_{1}$, $\left[\mathrm{T}\left(\mathrm{C}_{8}-\mathrm{F}\right) \mathrm{T}-\mathrm{Pd}-\mathrm{T}\left(\mathrm{C}_{8}-\mathrm{F}\right) \mathrm{T}\right]_{1}$ and $(\mathrm{d})\left[\mathrm{Hg}-\mathrm{T}\left(\mathrm{C}_{8}-\mathrm{F}\right) \mathrm{T}-\mathrm{Pt}-\mathrm{T}\left(\mathrm{C}_{8}-\mathrm{F}\right) \mathrm{T}-\mathrm{Hg}\right]_{1}$. For simplicity, the $\mathrm{PBu}_{3}$ ligands were modeled by $\mathrm{PMe}_{3}$ groups whereas the octyl chains on the fluorenyl ring were modeled by the methyl groups.

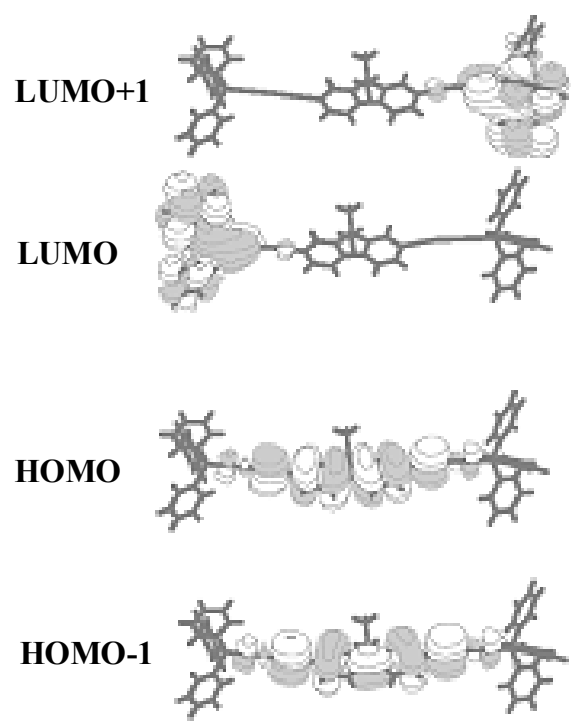

(a)
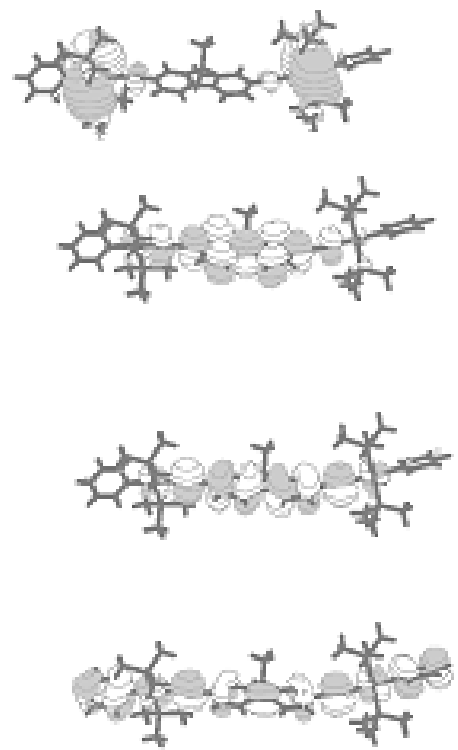

(b)
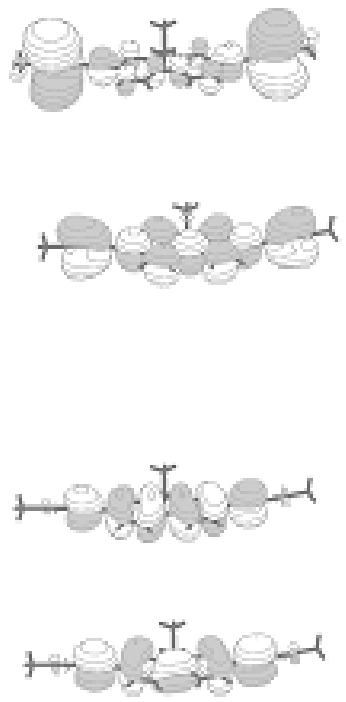

(c)

Figure S7. Spatial plots of the HOMOs and LUMOs for (a) $\left[\mathrm{Au}-\mathrm{T}\left(\mathrm{C}_{8}-\mathrm{F}\right) \mathrm{T}-\mathrm{Au}\right]_{1}$, (b) $\left[\mathrm{Pt}-\mathrm{T}\left(\mathrm{C}_{8}-\mathrm{F}\right) \mathrm{T}-\mathrm{Pt}\right]_{1}$ and $(\mathrm{c})\left[\mathrm{Hg}-\mathrm{T}\left(\mathrm{C}_{8}-\mathrm{F}\right) \mathrm{T}-\mathrm{Hg}\right]_{1}$. For simplicity, the $\mathrm{PBu}_{3}$ ligands were modeled by $\mathrm{PMe}_{3}$ groups whereas the alkyl chains on the fluorenyl ring were modeled by the methyl groups. 

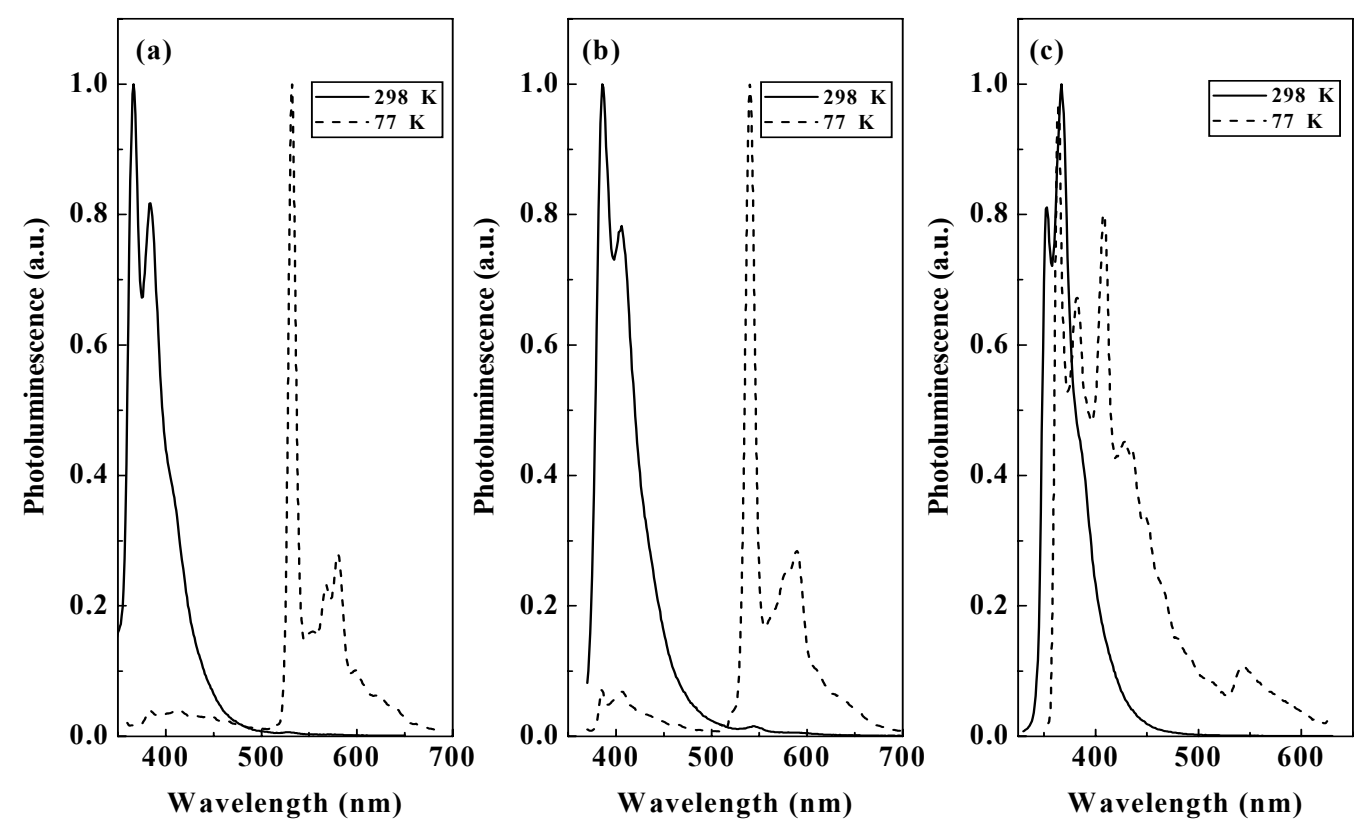

Figure S8. $\mathrm{PL}$ spectra at $298 \mathrm{~K}$ and $77 \mathrm{~K}$ in $\mathrm{CH}_{2} \mathrm{Cl}_{2}$ for (a) $\left[\mathrm{Au}-\mathrm{T}\left(\mathrm{C}_{8}-\mathrm{F}\right) \mathrm{T}-\mathrm{Au}\right]_{1}$, (b) $\left[\mathrm{Pt}-\mathrm{T}\left(\mathrm{C}_{8}-\mathrm{F}\right) \mathrm{T}-\mathrm{Pt}\right]_{1}$ and $(\mathrm{c})\left[\mathrm{Hg}-\mathrm{T}\left(\mathrm{C}_{8}-\mathrm{F}\right) \mathrm{T}-\mathrm{Hg}\right]_{1}$.
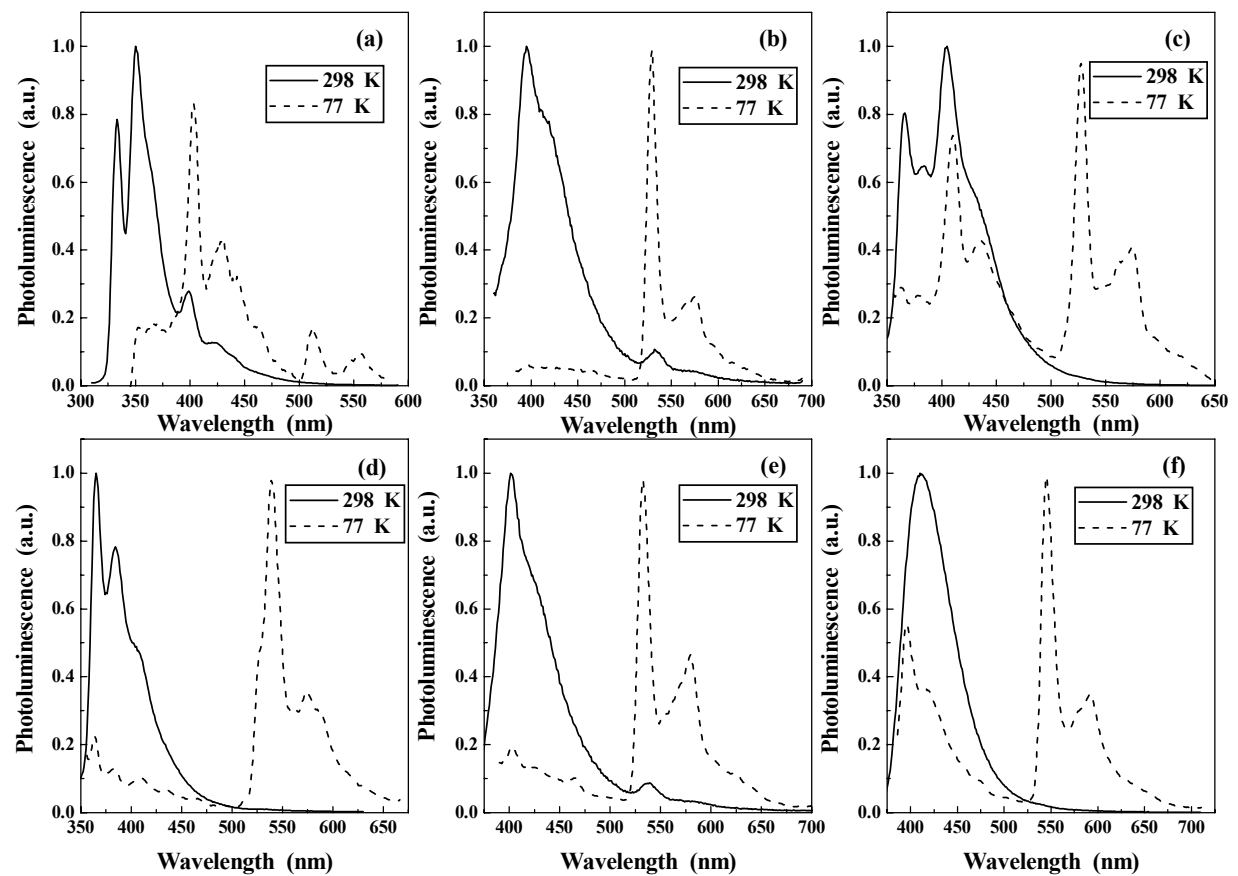

Figure S9. $\mathrm{PL}$ spectra at $298 \mathrm{~K}$ and $77 \mathrm{~K}$ in $\mathrm{CH}_{2} \mathrm{Cl}_{2}$ for (a) $\left[\mathrm{T}\left(\mathrm{C}_{8}-\mathrm{F}\right) \mathrm{T}-\mathrm{Hg}-\mathrm{T}\left(\mathrm{C}_{8}-\mathrm{F}\right) \mathrm{T}\right]_{1}$, (b) $\left[\mathrm{T}\left(\mathrm{C}_{8}-\mathrm{F}\right) \mathrm{T}-\mathrm{Pt}-\mathrm{T}\left(\mathrm{C}_{8}-\mathrm{F}\right) \mathrm{T}\right]_{1},(\mathrm{c})\left[\mathrm{Au}-\mathrm{T}\left(\mathrm{C}_{8}-\mathrm{F}\right) \mathrm{T}-\mathrm{Hg}-\mathrm{T}\left(\mathrm{C}_{8}-\mathrm{F}\right) \mathrm{T}-\mathrm{Au}\right]_{1},(\mathrm{~d})\left[\mathrm{Au}-\mathrm{T}\left(\mathrm{C}_{8}-\mathrm{F}\right) \mathrm{T}-\mathrm{Pt}-\mathrm{T}\left(\mathrm{C}_{8}-\mathrm{F}\right) \mathrm{T}-\mathrm{Au}\right]_{1}$, (e) $\left[\mathrm{Hg}-\mathrm{T}\left(\mathrm{C}_{8}-\mathrm{F}\right) \mathrm{T}-\mathrm{Pt}-\mathrm{T}\left(\mathrm{C}_{8}-\mathrm{F}\right) \mathrm{T}-\mathrm{Hg}\right]_{1}$ and (f) $\left[\mathrm{Pt}-\mathrm{T}\left(\mathrm{C}_{8}-\mathrm{F}\right) \mathrm{T}-\mathrm{Hg}-\mathrm{T}\left(\mathrm{C}_{8}-\mathrm{F}\right) \mathrm{T}-\mathrm{Pt}\right]_{1}$. 

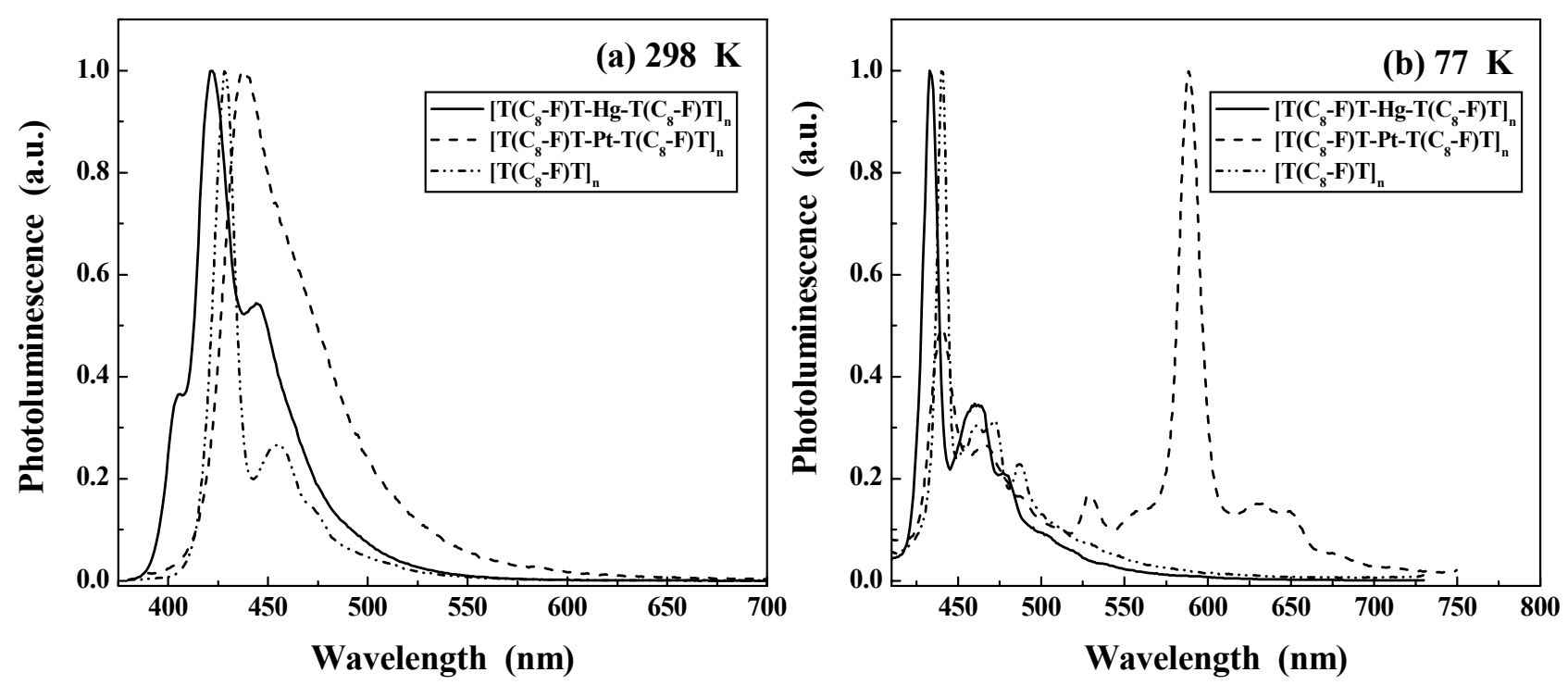

Figure S10. PL spectra for $\left[\mathrm{T}\left(\mathrm{C}_{8}-\mathrm{F}\right) \mathrm{T}-\mathrm{Hg}-\mathrm{T}\left(\mathrm{C}_{8}-\mathrm{F}\right) \mathrm{T}\right]_{\mathrm{n}},\left[\mathrm{T}\left(\mathrm{C}_{8}-\mathrm{F}\right) \mathrm{T}-\mathrm{Pt}-\mathrm{T}\left(\mathrm{C}_{8}-\mathrm{F}\right) \mathrm{T}\right]_{\mathrm{n}}$ and $\left[\mathrm{T}\left(\mathrm{C}_{8}-\mathrm{F}\right) \mathrm{T}\right]_{\mathrm{n}}$ in $\mathrm{CH}_{2} \mathrm{Cl}_{2}$ at (a) $298 \mathrm{~K}$ and (b) $77 \mathrm{~K}$.

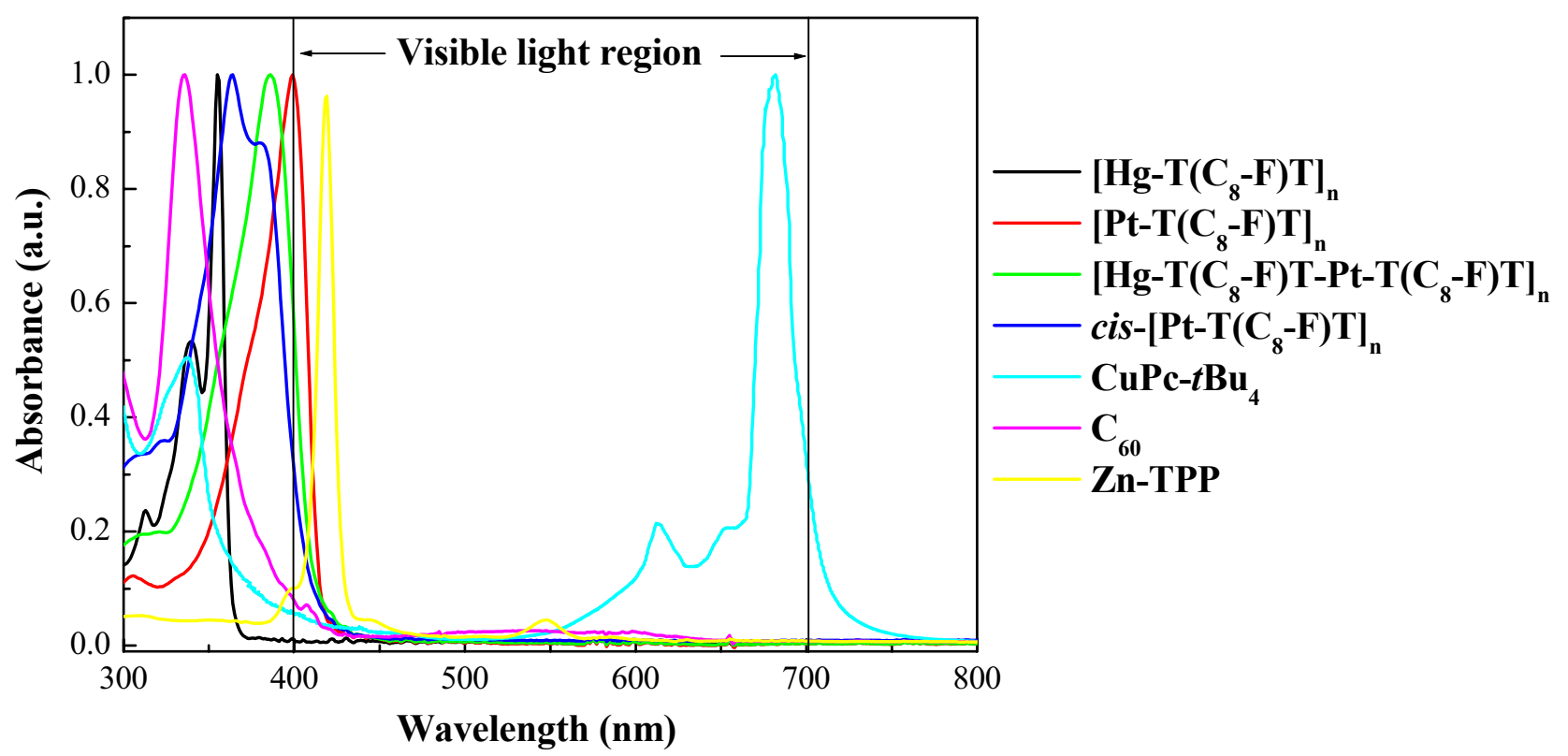

Figure S11. Comparison of the optical transparency windows of our polyplatinaynes with some state-of-the-art OPL materials. 

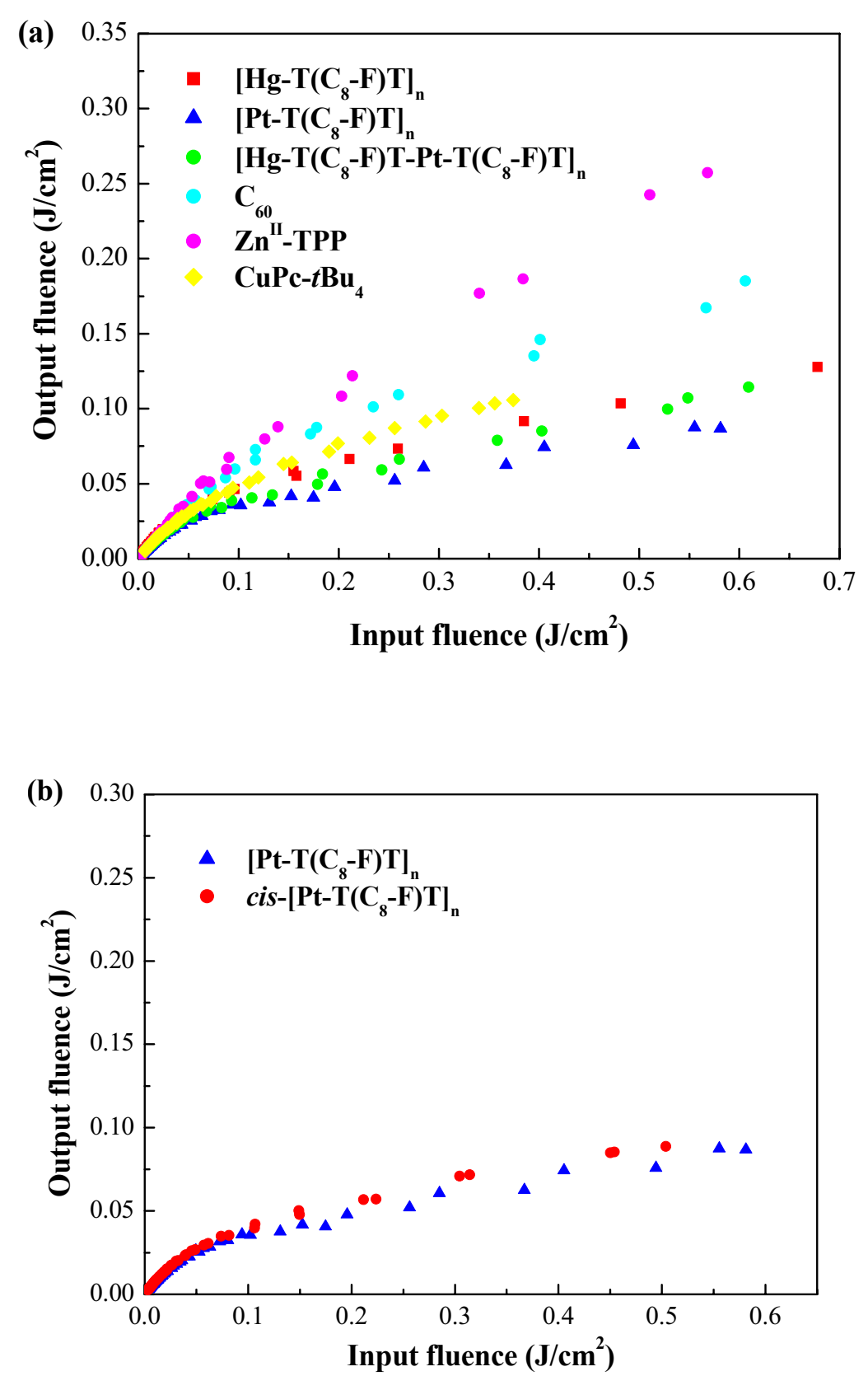

Figure S12. Comparison of the optical-limiting properties at the same linear transmittance of $92 \%$ for (a) the metallopolyynes versus state-of-the-art optical limiters and (b) $\left[\mathbf{P t}-\mathbf{T}\left(\mathbf{C}_{\mathbf{8}}-\mathbf{F}\right) \mathbf{T}\right]_{\mathbf{n}}$ versus cis-[Pt-T( $\left.\left.\mathbf{C}_{8}-\mathbf{F}\right) \mathbf{T}\right]_{n}$. 

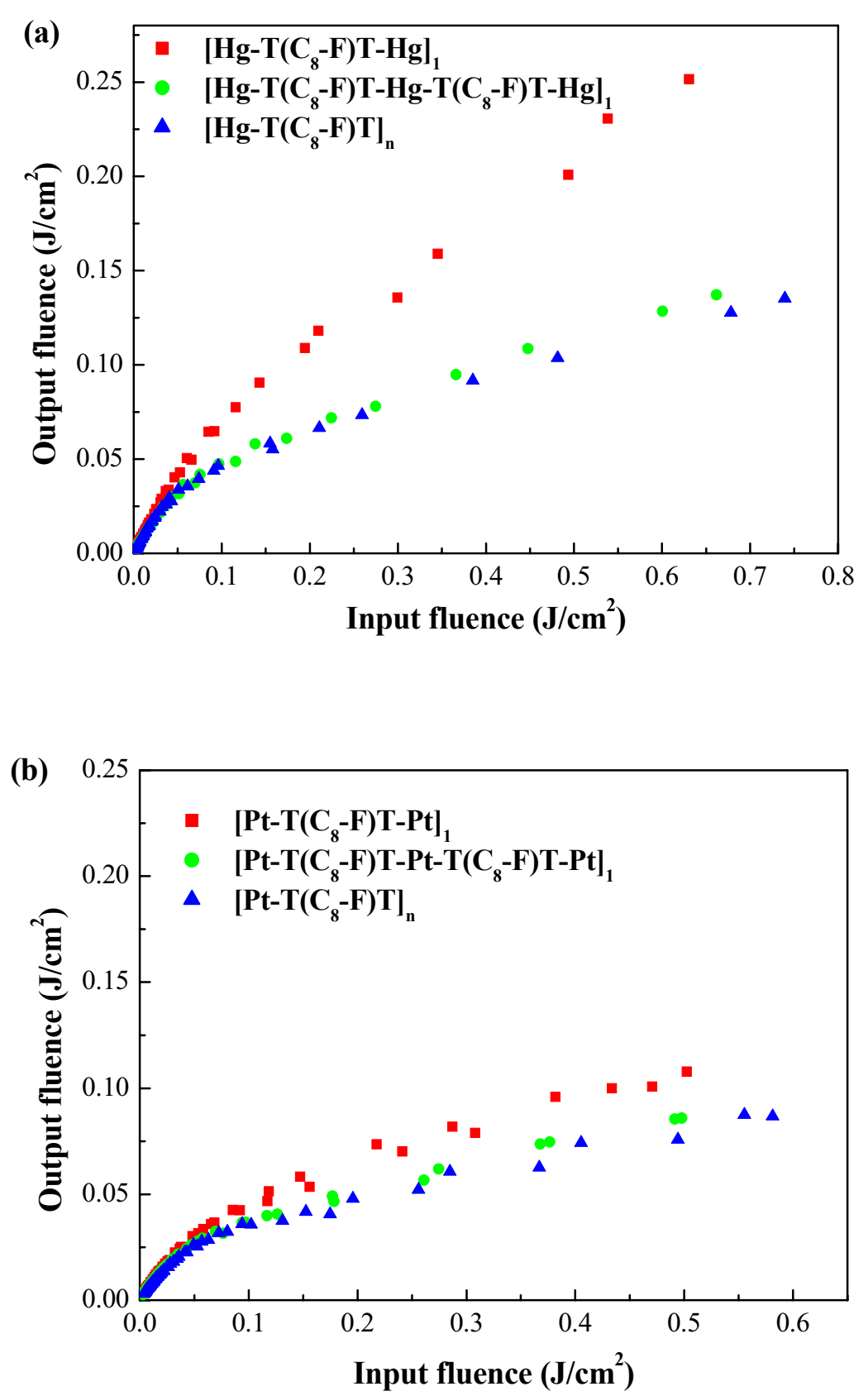

Figure S13. Comparison of the optical-limiting properties at the same linear transmittance of $92 \%$ for the (a) mercury-based alkynyl complexes and (b) platinum-based alkynyl complexes. 
Table S1. Crystallographic data for selected metallayne model complexes.

\begin{tabular}{|c|c|c|c|c|}
\hline & \multicolumn{4}{|c|}{$\left[\mathrm{Au}-\mathrm{T}\left(\mathrm{C}_{8}-\mathrm{F}\right) \mathrm{T}-\mathrm{Au}\right]_{1}\left[\mathrm{Hg}-\mathrm{T}\left(\mathrm{C}_{8}-\mathrm{F}\right) \mathrm{T}-\mathrm{Hg}\right]_{1}\left[\mathrm{~T}\left(\mathrm{C}_{8}-\mathrm{F}\right)-\mathrm{Pt}-\mathrm{T}\left(\mathrm{C}_{8}-\mathrm{F}\right) \mathrm{T}\right]_{1}\left[\mathrm{Hg}-\mathrm{T}\left(\mathrm{C}_{8}-\mathrm{F}\right) \mathrm{T}-\mathrm{Pt}-\mathrm{T}\left(\mathrm{C}_{8}-\mathrm{F}\right) \mathrm{T}-\mathrm{Hg}\right]_{1}$} \\
\hline formula & $\mathrm{C}_{70} \mathrm{H}_{72} \mathrm{Au}_{2} \mathrm{Cl}_{2} \mathrm{P}_{2}$ & $\mathrm{C}_{35} \mathrm{H}_{46} \mathrm{Hg}_{2}$ & $\mathrm{C}_{90} \mathrm{H}_{136} \mathrm{P}_{2} \mathrm{Pt}$ & $\mathrm{C}_{92} \mathrm{H}_{140} \mathrm{Hg}_{2} \mathrm{P}_{2} \mathrm{Pt}$ \\
\hline formula weight & 1440.05 & 867.90 & 1475.02 & 1904.25 \\
\hline crystal system & triclinic & monoclinic & Monoclinic & triclinic \\
\hline space group & $P \overline{1}$ & Cc & $P 2_{1} / c$ & $P \overline{1}$ \\
\hline$a(\AA)$ & $13.2656(11)$ & $21.8830(13)$ & $9.2061(7)$ & $9.5394(9)$ \\
\hline$b(\AA)$ & $16.5935(14)$ & $17.6803(13)$ & $26.541(2)$ & $12.9262(12)$ \\
\hline$c(\AA)$ & $16.9461(14)$ & $9.2053(6)$ & $17.6715(13)$ & $20.1042(18)$ \\
\hline$\alpha(\mathrm{deg})$ & $66.7900(10)$ & 90 & 90 & $77.171(2)$ \\
\hline$\beta(\operatorname{deg})$ & $67.593(2)$ & $107.840(2)$ & $94.703(2)$ & $78.359(2)$ \\
\hline$\gamma(\operatorname{deg})$ & $84.370(2)$ & 90 & 90 & $70.3490(10)$ \\
\hline$V\left(\AA^{3}\right)$ & $3163.5(5)$ & $3390.3(4)$ & $4303.3(6)$ & $2254.4(4)$ \\
\hline$Z$ & 2 & 4 & 2 & 1 \\
\hline$D_{\text {calcd }}\left(\mathrm{g} \mathrm{cm}^{-3}\right)$ & 1.512 & 1.700 & 1.138 & 1.403 \\
\hline$\mu\left(\mathrm{mm}^{-1}\right)$ & 4.806 & 9.065 & 1.708 & 5.020 \\
\hline$F(000)$ & 1428 & 1664 & 1568 & 960 \\
\hline$\theta$ range $(\mathrm{deg})$ & $1.66-25.00$ & $1.99-28.33$ & $1.92-25.00$ & $1.84-25.00$ \\
\hline reflections collected & 15385 & 10015 & 21190 & 10824 \\
\hline unique reflections & 10894 & 5992 & 7538 & 7674 \\
\hline$R_{\text {int }}$ & 0.0234 & 0.0419 & 0.0608 & 0.0369 \\
\hline observed reflections & 8160 & 4357 & 4297 & 4991 \\
\hline no. of parameters & 685 & 336 & 421 & 439 \\
\hline $\mathrm{R} 1, \mathrm{wR} 2[I>2.0 \sigma(I)][\mathrm{a}]$ & $0.0399,0.1023$ & $0.0317,0.0702$ & $0.0497,0.1166$ & $0.0588,0.1494$ \\
\hline R1, wR2 (all data) & $0.0598,0.1137$ & $0.0531,0.0795$ & $0.1059,0.1420$ & $0.0935,0.1734$ \\
\hline GoF on $F^{2}[\mathrm{~b}]$ & 1.025 & 0.888 & 0.969 & 0.976 \\
\hline
\end{tabular}

[a] R1 $=\Sigma\left\|F_{\mathrm{o}}|-| F_{\mathrm{c}}\right\| / \Sigma\left|F_{\mathrm{o}}\right| . \quad \mathrm{wR} 2=\left\{\Sigma\left[w\left(F_{\mathrm{o}}^{2}-F_{\mathrm{c}}{ }^{2}\right)^{2}\right] / \Sigma\left[w\left(F_{\mathrm{o}}{ }^{2}\right)^{2}\right]\right\}^{1 / 2}$.

$[\mathrm{b}] \mathrm{GoF}=\left[\left(\sum w\left|F_{\mathrm{o}}\right|-\left|F_{\mathrm{c}}\right|\right)^{2} /\left(N_{\mathrm{obs}}-N_{\text {param }}\right)\right]^{1 / 2}$. 
Table S2. Selected bond lengths $(\hat{A})$ and angles $\left(^{\circ}\right)$ for $\left[\mathrm{Au}-\mathrm{T}\left(\mathrm{C}_{8}-\mathrm{F}\right) \mathrm{T}-\mathrm{Au}\right]_{1},\left[\mathrm{Hg}-\mathrm{T}\left(\mathrm{C}_{8}-\mathrm{F}\right) \mathrm{T}-\mathrm{Hg}\right]_{1}$, $\left[\mathrm{T}\left(\mathrm{C}_{8}-\mathrm{F}\right) \mathrm{T}-\mathrm{Pt}-\mathrm{T}\left(\mathrm{C}_{8}-\mathrm{F}\right) \mathrm{T}\right]_{1}$ and $\left[\mathrm{Hg}-\mathrm{T}\left(\mathrm{C}_{8}-\mathrm{F}\right) \mathrm{T}-\mathrm{Pt}-\mathrm{T}\left(\mathrm{C}_{8}-\mathrm{F}\right) \mathrm{T}-\mathrm{Hg}\right]_{1}$.

\begin{tabular}{|c|c|c|c|}
\hline \multicolumn{2}{|c|}{$\left[\mathrm{Au}-\mathrm{T}\left(\mathrm{C}_{8}-\mathrm{F}\right) \mathrm{T}-\mathrm{Au}\right]_{1}$} & \multicolumn{2}{|c|}{$\left[\mathrm{Hg}-\mathrm{T}\left(\mathrm{C}_{8}-\mathrm{F}\right) \mathrm{T}-\mathrm{Hg}\right]_{1}$} \\
\hline $\mathrm{Au}(1)-\mathrm{P}(1)$ & $2.2756(10)$ & $\operatorname{Hg}(1)-\mathrm{C}(1)$ & $2.080(11)$ \\
\hline $\mathrm{Au}(2)-\mathrm{P}(2)$ & $2.2732(10)$ & $\operatorname{Hg}(1)-\mathrm{C}(2)$ & $2.045(8)$ \\
\hline $\mathrm{Au}(1)-\mathrm{C}(19)$ & $1.988(4)$ & $\mathrm{Hg}(2)-\mathrm{C}(18)$ & $2.044(9)$ \\
\hline $\operatorname{Au}(2)-C(51)$ & $2.002(4)$ & $\mathrm{Hg}(2)-\mathrm{C}(19)$ & $2.059(9)$ \\
\hline$C(19)-C(20)$ & $1.203(5)$ & $\mathrm{C}(2)-\mathrm{C}(3)$ & $1.186(11)$ \\
\hline$C(50)-C(51)$ & $1.187(6)$ & $C(17)-C(18)$ & $1.209(13)$ \\
\hline $\mathrm{C}(19)-\mathrm{Au}(1)-\mathrm{P}(1)$ & $178.4(13)$ & $\mathrm{C}(1)-\mathrm{Hg}(1)-\mathrm{C}(2)$ & $177.6(7)$ \\
\hline $\mathrm{C}(51)-\mathrm{Au}(2)-\mathrm{P}(2)$ & $179.66(17)$ & $\mathrm{Hg}(1)-\mathrm{C}(2)-\mathrm{C}(3)$ & $176.8(8)$ \\
\hline $\mathrm{Au}(1)-\mathrm{C}(19)-\mathrm{C}(20)$ & 172.4(4) & $\mathrm{C}(18)-\mathrm{Hg}(2)-\mathrm{C}(19)$ & $177.7(5)$ \\
\hline $\mathrm{Au}(2)-\mathrm{C}(51)-\mathrm{C}(50)$ & $176.7(5)$ & $\mathrm{Hg}(2)-\mathrm{C}(18)-\mathrm{C}(17)$ & $176.1(12)$ \\
\hline \multicolumn{2}{|c|}{$\left[\mathrm{T}\left(\mathrm{C}_{8}-\mathrm{F}\right) \mathrm{T}-\mathrm{Pt}-\mathrm{T}\left(\mathrm{C}_{8}-\mathrm{F}\right) \mathrm{T}\right]_{1}$} & \multicolumn{2}{|c|}{$\left[\mathrm{Hg}-\mathrm{T}\left(\mathrm{C}_{8}-\mathrm{F}\right) \mathrm{T}-\mathrm{Pt}-\mathrm{T}\left(\mathrm{C}_{8}-\mathrm{F}\right) \mathrm{T}-\mathrm{Hg}\right]_{1}$} \\
\hline $\mathrm{Pt}(1)-\mathrm{P}(1)$ & $2.312(2)$ & $\operatorname{Pt}(1)-\mathrm{P}(1)$ & $2.307(2)$ \\
\hline $\operatorname{Pt}(1)-C(13)$ & $2.012(7)$ & $\operatorname{Pt}(1)-C(13)$ & $1.993(9)$ \\
\hline $\mathrm{C}(13)-\mathrm{C}(14)$ & $1.199(9)$ & $C(13)-C(14)$ & $1.227(12)$ \\
\hline \multirow[t]{3}{*}{$C(44)-C(15)$} & $1.162(11)$ & $\mathrm{C}(44)-\mathrm{C}(45)$ & $1.248(14)$ \\
\hline & & $\operatorname{Hg}(1)-C(45)$ & $1.975(12)$ \\
\hline & & $\operatorname{Hg}(1)-C(46)$ & $2.005(14)$ \\
\hline$C(13 \mathrm{~A})-\mathrm{Pt}(1)-\mathrm{C}(13)$ & $179.996(1)$ & $C(13)-P t(1)-C(13 A)$ & $180.0(4)$ \\
\hline $\mathrm{C}(13)-\mathrm{Pt}(1)-\mathrm{P}(1 \mathrm{~A})$ & $92.1(2)$ & $\mathrm{P}(1)-\mathrm{Pt}(1)-\mathrm{P}(1 \mathrm{~A})$ & 180.0 \\
\hline $\mathrm{C}(13)-\mathrm{Pt}(1)-\mathrm{P}(1)$ & $88.0(2)$ & $\mathrm{C}(13)-\mathrm{Pt}(1)-\mathrm{P}(1)$ & $86.6(3)$ \\
\hline \multirow[t]{3}{*}{$\mathrm{P}(1 \mathrm{~A})-\mathrm{Pt}(1)-\mathrm{P}(1)$} & $179.998(1)$ & $\mathrm{C}(13)-\mathrm{Pt}(1)-\mathrm{P}(1 \mathrm{~A})$ & 93.4(3) \\
\hline & & $\mathrm{C}(44)-\mathrm{C}(45)-\mathrm{Hg}(1)$ & $173.0(11)$ \\
\hline & & $\mathrm{C}(45)-\mathrm{Hg}(1)-\mathrm{C}(46)$ & $175.3(6)$ \\
\hline
\end{tabular}


Table S3. Structural and thermal properties of the metallopolyynes.

\begin{tabular}{cccccc}
\hline polymer & $M_{\mathrm{n}}[\mathrm{a}]$ & $M_{\mathrm{w}}[\mathrm{a}]$ & $M_{\mathrm{w}} / M_{\mathrm{n}}$ & $T_{\mathrm{d}}$ (onset) $\left({ }^{\circ} \mathrm{C}\right)[\mathrm{b}]$ & color \\
\hline$\left[\mathrm{Hg}-\mathrm{T}\left(\mathrm{C}_{8}-\mathrm{F}\right) \mathrm{T}\right]_{\mathrm{n}}$ & 8880 & 13040 & 1.47 & $371 \pm 5$ & white \\
{$\left[\mathrm{Pt}-\mathrm{T}\left(\mathrm{C}_{8}-\mathrm{F}\right) \mathrm{T}\right]_{\mathrm{n}}$} & 30420 & 67700 & 2.23 & $368 \pm 5$ & off-white \\
cis-[Pt-T(C- $\left.\left.\mathrm{C}_{8}-\mathrm{F}\right) \mathrm{T}\right]_{\mathrm{n}}$ & 4030 & 4570 & 1.13 & $406 \pm 5$ & white \\
{$\left[\mathrm{Pd}-\mathrm{T}\left(\mathrm{C}_{8}-\mathrm{F}\right) \mathrm{T}\right]_{\mathrm{n}}$} & 5400 & 7530 & 1.39 & $338 \pm 5$ & yellow-orange \\
{$\left[\mathrm{Hg}-\mathrm{T}\left(\mathrm{C}_{8}-\mathrm{F}\right) \mathrm{T}-\mathrm{Pt}-\mathrm{T}\left(\mathrm{C}_{8}-\mathrm{F}\right) \mathrm{T}\right]_{\mathrm{n}}$} & 15700 & 29790 & 1.90 & $366 \pm 5$ & off-white \\
{$\left[\mathrm{Pd}-\mathrm{T}\left(\mathrm{C}_{8}-\mathrm{F}\right) \mathrm{T}-\mathrm{Pt}-\mathrm{T}\left(\mathrm{C}_{8}-\mathrm{F}\right) \mathrm{T}\right]_{\mathrm{n}}$} & 13280 & 25250 & 1.90 & $368 \pm 5$ & yellow-orange \\
{$\left[\mathrm{Pd}-\mathrm{T}\left(\mathrm{C}_{8}-\mathrm{F}\right) \mathrm{T}-\mathrm{Hg}-\mathrm{T}\left(\mathrm{C}_{8}-\mathrm{F}\right) \mathrm{T}\right]_{\mathrm{n}}$} & 4030 & 4520 & 1.12 & $368 \pm 5$ & yellow \\
{$\left[\mathrm{T}\left(\mathrm{C}_{8}-\mathrm{F}\right) \mathrm{T}-\mathrm{Hg}-\mathrm{T}\left(\mathrm{C}_{8}-\mathrm{F}\right) \mathrm{T}\right]_{\mathrm{n}}$} & 8030 & 11860 & 1.48 & $394 \pm 5$ & off-white \\
{$\left[\mathrm{T}\left(\mathrm{C}_{8}-\mathrm{F}\right) \mathrm{T}-\mathrm{Pt}-\mathrm{T}\left(\mathrm{C}_{8}-\mathrm{F}\right) \mathrm{T}\right]_{\mathrm{n}}$} & 8830 & 12360 & 1.40 & $406 \pm 5$ & pale-yellow \\
{$\left[\mathrm{T}\left(\mathrm{C}_{8}-\mathrm{F}\right) \mathrm{T}\right]_{\mathrm{n}}$} & 8850 & 12990 & 1.47 & $408 \pm 5$ & pale yellow \\
\hline
\end{tabular}

[a] Number-average $\left(M_{\mathrm{n}}\right)$ and weight-average $\left(M_{\mathrm{w}}\right)$ molecular weights were obtained by GPC against polystyrene standards. [b] By TGA under $\mathrm{N}_{2}$ at a heating rate of $20^{\circ} \mathrm{C} \mathrm{min}^{-1}$.

N.B. The GPC-estimated molecular weights should be cautiously viewed in light of the difficulties associated with utilizing GPC for rigid-rod polymers. GPC method does not really give absolute values of molecular weights but provides a measure of hydrodynamic volume. For rod-like polymers, appreciable differences in the hydrodynamic behavior from that of flexible polystyrene polymers would be anticipated. So, the values obtained by polystyrene calibration in GPC will likely experience a slight overestimation of the molecular weights. However, the absence of end-group NMR resonances corroborates the assertion that there is high degree of polymerization in most of these polymers. 